\title{
Mean value and Harnack inequalities for a certain class of degenerate parabolic equations
}

José C. Fernandes ${ }^{1}$

\section{Introduction}

In this paper we study the behavior of solutions of degenerate parabolic equations of the form

$$
v(x) u_{t}(x, t)=\sum_{i, j=1}^{n} D_{x_{i}}\left(a_{i j}(x, t) D_{x_{i}} u(x, t)\right)
$$

where the coefficients are measurable functions, and the coefficient matrix $A=\left(a_{i j}\right)$ is symmetric and satisfies

$$
w_{1}(x) \sum_{j=1}^{n} \lambda_{j}^{2}(x) \xi_{j}^{2} \leq \sum_{i, j=1}^{n} a_{i j}(x, t) \xi_{i} \xi_{j} \leq w_{2}(x) \sum_{j=1}^{n} \lambda_{j}^{2}(x) \xi_{j}^{2}
$$

for $\xi=\left(\xi_{1}, \ldots, \xi_{n}\right) \in \mathbf{R}^{n}$ and $(x, t) \in \Omega \times(a, b), \Omega$ a bounded open set in $\mathbf{R}^{n}$.

We are going to assume some conditions on the weights (non-negative functions that are locally integrable) $v, w_{1}, w_{2}$ and on the functions $\lambda_{j}, j=1, \ldots, n$, in order to be able to derive mean value and Harnack inequalities for solutions of (1.1). The assumptions on $\lambda_{j}$, which we list below, are the ones stated in [FL2].

1 This work was supported by FAPESP - Fundação de Amparo à Pesquisa do Estado de São Paulo- Brazil. 
(1.3) $\lambda_{1} \equiv 1, \lambda_{j}(x)=\lambda_{j}\left(x_{1}, \ldots, x_{j-1}\right), j=2, \ldots, n, x \in \mathbf{R}^{n}$

(1.4) Let $\Pi=\left\{x \in \mathbf{R}^{n}: \Pi x_{k}=0\right\}$. Then $\lambda_{j} \in C\left(\mathbf{R}^{n}\right) \cap C^{1}\left(\mathbf{R}^{n} \backslash \Pi\right)$ and $0<\lambda_{j}(x) \leq \Lambda, x \in \mathbf{R}^{n} \backslash \Pi, j=1, \ldots, n$.

(1.5) $\lambda_{j}\left(x_{1}, \ldots, x_{i}, \ldots, x_{j-1}\right)=\lambda_{j}\left(x_{1}, \ldots,-x_{i}, \ldots, x_{j-1}\right)$, for $j=2, \ldots, n$ and $i=1, \ldots, j-1$.

(1.6) There is a family of $n(n-1) / 2$ non-negative numbers $\varrho_{j, i}$ such that $0 \leq x_{i}\left(D_{x_{i}} \lambda_{j}\right)(x) \leq \varrho_{j, i} \lambda_{j}(x)$, for $2 \leq j \leq n, 1 \leq i \leq j-1$ and all $x \in R^{n} \backslash \Pi$.

Denote $\Gamma=\Omega \times(a, b)$ and define $H=H(\Gamma)$ to be the closure of $\operatorname{Lip}(\Gamma)$ under the norm

$$
\text { (1.7) } \begin{aligned}
\|u\|^{2} & =\iint_{\Gamma} u^{2}(x, t)\left(v(x)+w_{2}(x)\right) d x d t \\
& +\iint_{\Gamma}\left|\nabla_{\lambda} u(x, t)\right|^{2} w_{2}(x) d x d t+\iint_{\Gamma} u_{t}^{2}(x, t) v(x) d x d t,
\end{aligned}
$$

where $\nabla_{\lambda} u=\left(\lambda_{1} D_{x_{1}} u, \ldots, \lambda_{n} D_{x_{n}} u\right)$. Thus, $H(\Gamma)$ is the collection of all $(n+2)$ triples $(u, \beta, B)$ such that there exists $u_{k} \in \operatorname{Lip}(\Gamma)$ with $u_{k} \rightarrow u, \nabla_{\lambda} u_{k} \rightarrow \beta$, $\left(u_{k}\right)_{t} \rightarrow B$, the convergence being in the appropriate $L^{2}$ space. We need to verify that $\beta$ is uniquely determined and for this it is enough to show that for every $F \in C_{0}^{\infty}(\Gamma)$,

$$
\int_{\Gamma} u \nabla_{\lambda} F=-\int_{\Gamma} \beta F
$$

In order to prove this, note that since $u \in H$, there exists $\left\{u_{k}\right\} \subset \operatorname{Lip}(\Gamma)$ such that $u_{k} \rightarrow u$ in $H$. Then, by (1.3),

$$
\int_{\Gamma} u_{k} \lambda_{i} \frac{\partial F}{\partial x_{i}}=-\int_{\Gamma} \frac{\partial}{\partial x_{i}}\left(u_{k} \lambda_{i}\right) F=-\int_{\Gamma} \lambda_{i} \frac{\partial u_{k}}{\partial x_{i}} F
$$

Therefore,

$$
\int_{\Gamma} u_{k} \nabla_{\lambda} F=-\int_{\Gamma}\left(\nabla_{\lambda} u_{k}\right) F
$$

By Schwarz's inequality and assuming that $w_{2}^{-1} \in L_{\mathrm{loc}}^{1}$, 


$$
\begin{aligned}
\left|\int_{\Gamma} u_{k} \nabla_{\lambda} F-\int_{\Gamma} u \nabla_{\lambda} F\right| & \leq \int_{\Gamma}\left|u_{k}-u\right| w_{2}^{1 / 2}\left|\nabla_{\lambda} F\right| w_{2}^{-1 / 2} \\
& \leq\left\|u_{k}-u\right\|_{L_{w_{2}}^{2}}\left(\int_{\Gamma}\left|\nabla_{\lambda} F\right|^{2} w_{2}^{-1}\right)^{1 / 2} \\
& \leq c\left\|u_{k}-u\right\|_{L_{w_{2}}^{2}} .
\end{aligned}
$$

Hence,

$$
\int_{\Gamma} u_{k} \nabla_{\lambda} F \rightarrow \int_{\Gamma} u \nabla_{\lambda} F
$$

and similarly we can show

$$
\int_{\Gamma}\left(\nabla_{\lambda} u_{k}\right) F \rightarrow \int_{\Omega} \beta F
$$

In the same way we prove $B$ is uniquely determined, if $v^{-1} \in L_{\mathrm{loc}}^{1}$. We also define $H_{0}(\Gamma)$ to be the closure of $\operatorname{Lip}_{0}(\Gamma)$, Lipschitz functions with compact support in $\Gamma$, under the norm defined in (1.7). It is easy to see that the bilinear form $b$ on $\operatorname{Lip}(\Gamma) \cap H(\Gamma)$ defined by

$$
b(u, \phi)=\iint_{\Gamma}\left\{u_{t} \phi v+\langle A \nabla u, \nabla \phi\rangle\right\} d x d t
$$

can be continued to all of $H(\Gamma)$ (here and in the rest of the paper the vector $\nabla u$ is understood to be the vector $\left(\frac{1}{\lambda_{1}} \beta_{1}, \ldots, \frac{1}{\lambda_{n}} \beta_{n}\right)$ where $\left.\nabla_{\lambda} u=\left(\beta_{1}, \ldots, \beta_{n}\right)\right)$. We say $u \in H(\Gamma)$ is a solution of $(1.1)$ if $b(u, \phi)=0$ for any $\phi \in H_{0}$; $u \in H(\Gamma)$ is a subsolution if $b(u, \phi) \leq 0$ for any $\phi \in H_{0}(\Gamma), \phi$ positive in the $H$-sense, i.e., $\phi$ can be approximated in $H(\Gamma)$ by positive functions with compact support in $\Gamma ; u \in H(\Gamma)$ is a supersolution if $b(u, \phi) \leq 0$ for any $\phi \in H_{0}$, $\phi$ positive in the $H$-sense.

We also define $\tilde{H}=\tilde{H}(\Omega)$ to be the closure of $\operatorname{Lip}(\Omega)$ under the norm

$$
\|u\|^{2}=\int_{\Gamma} u^{2}(x)\left(v(x)+w_{2}(x)\right) d x+\int_{\Gamma}\left|\nabla_{\lambda} u(x)\right|^{2} w_{2}(x) d x,
$$

and $\tilde{H}_{0}(\Omega)$ to be the closure of $\operatorname{Lip}_{0}(\Omega)$ under the norm defined above.

Next we will define a natural distance (associated with the functions $\lambda_{j}$, $j=1, \ldots, n)$ and state some of its properties. This metric was first introduced by $[\mathrm{FL} 1]$.

A vector $v \in \mathbf{R}^{n}$ is called a $\lambda$-subunit vector at a point $x$ if $\langle v, \xi\rangle^{2} \leq \Sigma \lambda_{j}^{2}(x) \xi_{j}^{2}$, for every $\xi \in \mathbf{R}^{n}$. An absolutely continuous curve $\gamma:[0, T] \rightarrow \mathbf{R}^{n}$ is called a $\lambda$-subunit curve if $\dot{\gamma}(t)$ is a $\lambda$-subunit vector at $\gamma(t)$ for a.e. $t \in[0, T]$.

For any $x, y \in \mathbf{R}^{n}$ we define $d: \mathbf{R}^{n} \times \mathbf{R}^{n} \rightarrow \mathbf{R}^{+}$by 
$\begin{aligned} d(x, y)=\inf \left\{\boldsymbol{T} \in \mathbf{R}^{+}:\right. & \text {there exists a } \lambda \text {-subunit curve } \gamma:|0, T| \rightarrow \mathbf{R}^{n} \\ & \text { with } \gamma(0)=x, \gamma(T)=y\} .\end{aligned}$

One can check that this is a well-defined metric. There is a quasi-metric $\delta$ (a function $\delta: \mathbf{R}^{n} \times \mathbf{R}^{n} \rightarrow \mathbf{R}^{+}$is called a quasi-metric if there exists $M \geq 1$ such that $\delta(x, y) \leq M\{\delta(x, z)+\delta(z, y)\}$ for all $\left.x, y, z \in \mathbf{R}^{n}\right)$ equivalent to $d$, and sometimes easier to work with than $d$ (see [FL2]). If $x \in \mathbf{R}^{n}$ and $t \in \mathbf{R}$ put $H_{0}(x, t)=x$ and $H_{k+1}(x, t)=H_{k}(x, t)+t \lambda_{k+1}\left(H_{k}(x, t)\right) e_{k+1}$ for $k=0, \ldots$, $n-1$, where $\left\{e_{k}\right\}$ is the standard basis in $\mathbf{R}^{n}$. Define $\varphi_{j}\left(x^{*},.\right)=\left(F_{j}\left(x^{*}, .\right)\right)^{-1}$, the inverse function of $F_{j}\left(x^{*},.\right)$, where $F_{j}(x, s)=s \lambda_{j}\left(H_{j-1}(x, s)\right)$, for $j=1$, $\ldots, n$ and $x^{*}=\left(\left|x_{1}\right|, \ldots,\left|x_{n}\right|\right)$.

We define $\delta: \mathbf{R}^{n} \times \mathbf{R}^{n} \rightarrow \mathbf{R}^{+}$as

$$
\delta(x, y)=\operatorname{Max}_{\mathrm{j}=1, \ldots, n} \varphi_{j}\left(x^{*},\left|x_{j}-y_{j}\right|\right) .
$$

Note that

(1.8) $\delta(x, y)<s$ is equivalent to $\left|x_{j}-y_{j}\right|<F_{j}\left(x^{*}, s\right), 1 \leq j \leq n$.

In (1.9), (1.10), (1.11) below we state some basic facts concerning $\delta$ and $d$ (see also [FL2].

(1.9) There exists $a \geq 1$ such that for any $x, y \in \mathbf{R}^{n}$,

$$
a^{-1} \leq \frac{d(x, y)}{\delta(x, y)} \leq a
$$

Consequently, $\delta$ is a quasi-metric with $\left.\delta(x, y) \leq a^{2} \mid \delta(x, y)+\delta(z, y)\right\rfloor$ and $\delta(x, y) \leq a^{2} \delta(y, x)$.

(1.10) For any $x \in \mathbf{R}^{n}, s>0$ and $\left.\theta \in\right] 0,1 \mid$

$$
\theta^{G_{j}} \leq \frac{F_{j}\left(x^{*}, \theta s\right)}{F_{j}\left(x^{*}, s\right)} \leq \theta
$$

where $G_{1}=1$ and $G_{j}=1+\Sigma_{l=1}^{j-1} G_{l} \varrho_{j, l}$, for $j=2, \ldots, n$.

(1.11) We denote $S(x, r)=\left\{y \in \mathbf{R}^{n}: d(x, y)<r\right\}$ and $Q(x, r)=\left\{y \in \mathbf{R}^{n}\right.$ : $\delta(x, y)<r\}$ and we will call $S(x, r)$ a $d$-ball and $Q(x, r)$ a $\delta$-ball. Note that there is a constant $A>1$ such that $|S(x, 2 r)| \leq A|S(x, r)|$ and $|Q(x, 2 r)| \leq A|Q(x, r)|$, where $|\cdot|$ denotes Lebesgue measure. Also, by (1.8), $|Q(x, r)|=\prod_{j=1}^{n} F_{j}\left(x^{*}, r\right)$. If $Q=Q(x, r)$, we write $r=r(Q)$. 
In general we say that a non-negative and locally integrable function $w(x)$ is a doubling weight $(w \in D)$ if there exists a constant $A>1$ such that $w(2 Q)$ $\leq A w(Q)$ for any $\delta$-ball $Q$, where $2 Q=Q(x, 2 r)$, if $Q=Q(x, r)$ and

$$
w(Q)=\int_{Q} w(x) d x
$$

(1.12) If $w \in D$ then there exists $\alpha>0$ such that, for all $r>0, \theta \in] 0,1]$, and $x \in R^{n}, w(Q(x, \theta r)) \geq \theta^{\alpha} w(Q(x, r))$.

Given $1<p<\infty$, we say $w \in A_{p}$ if there is a constant $c>0$ such that for all $\delta$-balls $Q$ in $\mathbf{R}^{n}$.

$$
\left(\frac{1}{|Q|} \int_{Q} w(x) d x\right)\left(\frac{1}{|Q|} \int_{Q} w(x)^{-1 / p-1} d x\right)^{p-1} \leq c
$$

Note that if we have the $A_{p}$ condition with respect to $\delta$, we have the same condition holding for the metric $d$, i.e. (1.13) holds with $Q$ replaced by $S$ (using doubling and the equivalence between $d$ and $\delta$ ). If $v$ is a weight, $w \in A_{p}(v)$ means an analogous inequality holds with $d x$ and $|Q|$ replaced by $v(x) d x$ and $v(Q)$, respectively. We use the notation $A_{\infty}(v)=\cup_{p>1} A_{p}(v)$. The theory of weights in homogeneous spaces was studied by A. P. Calderón in $[C]$ and frequently we refer to this paper.

If $x, y \in \mathbf{R}^{n}$, we shall denote by $H(t, x, y)=\left(H_{1}(t, x, y), \ldots, H_{n}(t, x, y)\right)$ the solution at time $t$ of the Cauchy problem $\dot{H}_{j}(., x, y)=y_{j} \lambda_{j}(H(., x, y))$, $H_{j}(0, x, y)=x_{j}, j=1, \ldots, n$.

Given $\alpha=\left(\alpha_{1}, \ldots, \alpha_{n}\right), \epsilon=\left(\epsilon_{1}, \ldots, \epsilon_{n}\right)$ with $0<\epsilon_{j}<\alpha_{j}, j=1, \ldots, n$, we denote $\Delta_{\epsilon}^{\alpha}=\left\{y \in \mathbf{R}^{n}: \epsilon_{j} \leq y_{j} \leq \alpha_{j}, j=1, \ldots, n\right\}$. If $\sigma \in\{-1,1\}^{n}$, we put $T_{\sigma} y=\left(\sigma_{1} y_{1}, \ldots, \sigma_{n} y_{n}\right), Q^{\sigma}(x, r)=\left\{y \in Q(x, r): \sigma_{j}\left(y_{j}-x_{j}\right) \geq 0, j=1\right.$, $\ldots, n\}$ and $\Delta_{\epsilon}^{\alpha}(\sigma)=T_{\sigma}\left(\Delta_{\epsilon}^{\alpha}\right)$.

Now we can state two results proved in [FS].

Let $\gamma \in] 0,1 \mid$ and $\sigma \in\{-1,1\}^{n}$ be fixed. Then there exists $\epsilon, \alpha \in \mathbf{R}^{n}$ as above such that, for all $r>0$ and $x \in \mathbf{R}^{n}$,

$$
\left|H\left(r, x, \Delta_{\epsilon}^{\alpha}(\sigma)\right) \bigcap Q^{\sigma}(x, r)\right| \geq(1-\gamma)\left|Q^{\sigma}(x, r)\right|,
$$

where $H\left(r, x, \Delta_{\epsilon}^{\alpha}(\sigma)\right)=\left\{H(x, r, y): y \in \Delta_{\epsilon}^{\alpha}(\sigma)\right\}$.

Also, there are positive constants $c_{1}, c_{2}$ depending only on $\epsilon, \alpha$ and $\varrho_{j, i}$ such that 


$$
c_{1}|S(x, r)| \leq \prod \int_{0}^{r} \lambda_{j}(H(t, x, y)) d t \leq c_{2}|S(x, r)|
$$

for each $x \in \mathbf{R}^{n}, r>0$ and $y \in \Delta_{\epsilon}^{\alpha}(\sigma)$.

If $q \geq 2$, we say that Sobolev inequality holds for $w_{1}, n_{2}$ for any $u \in \tilde{H}_{0}(Q), Q$ a $\delta$-ball in $\mathbf{R}^{n}$,

$$
\left(\frac{1}{w_{2}(Q)} \int_{Q}|u|^{q} w_{2} d x\right)^{1 / q} \leq \operatorname{cr}(Q)\left(\frac{1}{w_{1}(Q)} \int_{Q}\left|\nabla_{\lambda} u\right|^{2} w_{1} d x\right)^{1 / 2}
$$

Given $q \geq 2$, we say the Poincare inequality holds for $w_{1}, w_{2}$ and $\mu$ if there are constant $c>0$ and $a>0$ (see (1.9)) such that for any $\delta$ ball $Q$ and every $u \in \tilde{H}\left(a^{2} Q\right)$ we have

$$
\begin{aligned}
\left(\frac{1}{w_{2}(Q)} \int_{Q}\left|u-a v_{\mu, Q} u\right|^{q} w_{2} d x\right)^{1 / q} & \leq \\
& \leq \operatorname{cr}(Q)\left(\frac{1}{w_{1}(Q)} \int_{a^{2} Q}\left|\nabla_{\lambda} u\right|^{2} w_{1} d x\right)^{1 / 2},
\end{aligned}
$$

where $a v_{\mu, Q} u=\frac{1}{\mu(Q)} \int_{Q} u d \mu$ and $a^{2} Q=Q\left(x, a^{2} r\right)$ if $Q=Q(x, r)$.

The reason that we have $a^{2} Q$ on the right side of (1.17) is that we do not have a Kohn type argument (see also $\mid \mathrm{J}]$ ) for the quasi-metric $\delta$. In the $d$-metric, we can state (1.17) with equal balls on both sides. For the metric $\delta$, however, we have convenient cut-off functions (see [FL1]) that are important in order to get Caccioppoli estimates for solutions of (1.1) (see C.1), (C.2) and (C.3)). This explains the reason that we work with $\delta$ instead of $d$.

We can now state our main results.

Theorem A (Harnack's inequality).

\section{Suppose that:}

(i) $v, w_{1}, w_{2} \in A_{2}$,

(ii) the Poincare inequality holds for $w_{1}, w_{2}$ and $w_{1}, v$ with $\mu=1$ and some

$$
q>2 \text {, }
$$

(iii) $w_{2} v^{-1} \in A_{\infty}(v)$.

If $u$ is a non-negative solution of (1.1) in the cylinder $R=Q\left(x_{0}, \alpha\right) \times\left(t_{0}-\beta\right.$, $\left.t_{0}+\beta\right)$, then 
ess $\sup _{R^{-}} u \leq c_{1} \exp \left\{c_{2}\left[\alpha^{-2} \beta \Lambda\left(Q\left(x_{0}, \alpha\right)\right)+\alpha^{2} \beta^{-1}\left(\lambda\left(Q\left(x_{0}, \alpha\right)\right)\right)^{-1}\right]\right\} \operatorname{ess} \inf _{R^{+}} u$, where $R^{-}=Q\left(x_{0}, \alpha / 2\right) \times\left(t_{0}-3 \beta / 4, t_{0}-\beta / 4\right), R^{+}=Q\left(x_{0}, \alpha / 2\right) \times\left(t_{0}+\beta / 4\right.$, $\left.t_{0}+\beta\right), \Lambda(Q)=w_{2}(Q) / v(Q), \lambda(Q)=w_{1}(Q) / v(Q)$, for a $\delta$-ball $Q$. Here the constants $c_{1}, c_{2}$ depend only on the constants which arise in (i), (ii), (iii).

We write

$$
\iint_{R} f(x, t) m(x, t) d x d t=\iint_{R} f(x, t) m(x, t) d x d t / \iint_{R} m(x, t) d x d t .
$$

Theorem B (Mean value inequality). Assume that hypotheses (i), (ii), (iii) of Theorem A hold. Let $0<p<\infty, \alpha, \beta>0, \alpha / 2<\alpha^{\prime}<\alpha, \beta / 2<\beta^{\prime}<\beta$ and let $Q=Q\left(x_{0}, \alpha\right), Q^{\prime}=Q\left(x_{0}, \alpha^{\prime}\right)$ and $R=Q \times\left(t_{0}-\beta, t_{0}+\beta\right)$, $R_{+}^{\prime}=Q^{\prime} \times\left(t_{0}-\beta^{\prime}, t_{0}+\beta\right)$. If $u$ is a solution of $(1.1)$ in $R$, then $u$ is bounded in $R_{+}^{\prime}$ and

ess $\sup _{R_{+}^{\prime}}|u|^{p}$

$$
\leq D\left(\alpha^{2} \beta^{-1} \lambda(Q)^{-1}+1\right)^{1 /(h-1)}\left(\alpha^{-2} \beta \Lambda(Q)+1\right)^{h /(h-1)} \iint_{R}|u|^{p}\left(\alpha^{-2} \beta w_{2}+v\right) d x d t,
$$

where $D \leq C^{1 /(h-1)}$ if $p \geq 2$, and $D \leq c^{\log (3 / p)} C^{c}$ if $0<p<2$, and $C=c \frac{\alpha^{2+b} \beta}{\left(\alpha-\alpha^{\prime}\right)^{2+b}\left(\beta-\beta^{\prime}\right)}$. Here $h>1, c>0$ and $b>0$ are constants which are independent of $u, p, \alpha, \alpha^{\prime}, \beta, \beta^{\prime}$.

The organization of the paper is as follows. In Section 2 we prove the following Sobolev interpolation inequality:

Theorem D: Let $w_{1}, w_{2}$ be doubling weights, $v \in A_{2}$ ana' suppose (1.17) holds with $w_{1}, w_{2}, \mu=1$ and some $q>2$. If $w_{2} v^{-1} \in A_{\infty}(v)$ then there exists $h>$ 1 and constants $c>0, b>0$ such that for every $\epsilon$ satisfying $0<\epsilon \leq 1$,

$$
\begin{aligned}
\frac{1}{w_{2}(Q)} \int_{Q}|u|^{2 h} w_{2} d x & \\
& \leq c \epsilon^{-b}\left(\frac{1}{v(Q)} \int_{(1+\epsilon) Q} u^{2} v d x\right)^{h-1} \\
& \times\left(\frac{r(Q)^{2}}{w_{1}(Q)} \int_{(1+\epsilon) Q}^{:}\left|\nabla_{\lambda} u\right|^{2} w_{1} d x+\right. \\
& +\frac{1}{v(Q)} \int_{(1+\epsilon) Q}^{\left.u^{2} v d x\right)}
\end{aligned}
$$

for all $u \in \tilde{H}((1+\epsilon) Q)$. 
In section 3 we prove Theorem B. First we show, for $p \geq 2$, the following mean value inequality for subsolutions of (1.1):

$\left({ }^{*}\right)$ ess $\sup _{R_{+}^{\prime}} u_{+}^{p} \leq$

$\left(p^{2} C\right)^{\frac{h}{h-1}}\left(1+\alpha^{2} \beta^{-1} \lambda(Q)^{-1}\right)^{1 /(h-1)}\left(1+\alpha^{-2} \beta \Lambda(Q)\right)^{h /(h-1)} \iint_{R} u_{+}^{p}\left(\alpha^{-2} \beta w_{2}+v\right) d x d t$,

where $C$ is as in Theorem $\mathrm{B}$ and $u_{+}=\max \{u, 0\}$. This inequality is less precise than the one we will eventually obtain because of the presence of the factor $p^{2}$ on the right. In order to prove the above inequality we apply Theorem D to the function $H_{M}(u(., r))$ where

$$
H_{M}(s)=\left\{\begin{array}{l}
s^{p / 2} \text { if } s \in[0, M] \\
M^{p / 2}+\frac{p}{2} M^{(p-2) / 2}(s-M) \text { if } s \geq M \\
0 \text { if } s<0
\end{array}\right.
$$

and therefore $H_{M}(u(., \tau))$ is an element of $\tilde{H}\left(Q\left(x_{0}, \alpha\right)\right.$ for a.e. $\tau \in\left(t_{0}-\beta^{\prime}\right.$, $\left.t_{0}+\beta\right)$. The first idea would be to apply Theorem D to the function $u_{+}^{p / 2}($. , $\tau)$ but at this point we do not know if $u_{+}^{p / 2}(., \tau)$ belongs to $\tilde{H}\left(Q\left(x_{0}, \alpha\right)\right.$. Hence we have to work with $H_{M}(u)$, and in order to proceed with the proof of $\left({ }^{*}\right)$ we show the following Caccioppolli inequality for $H_{M}(u)$.

(C.1) Let $2 \leq p<\infty$ and $u$ be a subsolution of (1.1) in $R$. Let $w_{2} \in A_{2}$ and $\alpha, \alpha^{\prime}, \beta, \beta^{\prime}$ satisfy $\alpha / 2<\alpha^{\prime}<\alpha, \beta / 2<\beta^{\prime}<\beta$. Then

$$
\begin{aligned}
& \operatorname{ess} \sup _{\tau \in\left(t_{0}-\beta^{\prime}, t_{0}+\beta\right)} \int_{Q} H_{M}(u(x, r))^{2} v(x) d x \\
& \quad+\iint_{R^{\prime}}\left|\nabla_{\lambda}\left(H_{M}(u)\right)\right|^{2} w_{1}(x) d x d t \\
& \quad \leq c \iint_{R} u^{2} H_{M}^{\prime}(u)^{2}\left(\frac{w_{2}}{\left(\alpha-\overline{\alpha^{\prime}}\right)^{2}}+\frac{v}{\beta-\beta^{\prime}}\right) d x d t
\end{aligned}
$$

with $c$ independent of all parameters.

The next step is to apply $\left({ }^{*}\right)$ for $p=2$ to deduce that $u_{+}$is locally bounded. This fact allow us to apply Theorem $\mathrm{D}$ to the function $u_{+}^{p / 2}(., \tau)$ for a.e. $\tau \in\left(t_{0}-\beta^{\prime}, t_{0}+\beta\right)$. The Caccioppoli inequality we can deduce from (C.1) 
for the function $u_{+}^{p / 2}$ is not precise enough since it will have a factor $p^{2}$ in the right hand side (note that $u H_{M}^{\prime}(u) \leq p u_{+}^{p / 2} / 2$ ) and this is the term we want to eliminate from $(*)$. But with a different test function from the one used in the proof of (C.1), namely, $\phi(x, t)=\eta^{2} g(u) \chi\left(t, \tau_{1}, \tau_{2}\right)$ where

$$
g(s)=\left\{\begin{aligned}
s^{p-1} & \text { if } \quad s \in[0, M], \\
M^{p-2} s & \text { if } \quad s \geq M, \\
0 & \text { if } \quad s<0
\end{aligned}\right.
$$

and $\eta$ is a convenient $C^{\infty}$ function with compact support, we can deduce the following Caccioppoli inequality for subsolutions of (1.1):

(C.2) Let $2 \leq p<\infty$ and $u$ be a subsolution of (1.1) in $R$. Let $w_{2} \in A_{2}$ and $\alpha, \alpha^{\prime}, \beta, \beta^{\prime}$ satisfy $\alpha / 2<\alpha^{\prime}<\alpha, \beta / 2<\beta^{\prime}<\beta$. Then

$$
\begin{aligned}
& \text { ess } \sup _{\tau \in\left(t_{0}-\beta^{\prime}, t_{0}+\beta\right)} \int_{Q} u_{+}(x, \tau)^{p} v(x) d x+\iint_{R_{+}^{\prime}}\left|\nabla_{\lambda} u_{+}^{p / 2}\right|^{2} w_{1}(x) d x d t \\
& \leq c \iint_{R} u_{+}^{p}\left(\frac{w_{2}}{\left(\alpha-\alpha^{\prime}\right)^{2}}+\frac{v}{\beta-\beta^{\prime}}\right) d x d t
\end{aligned}
$$

with $c$ independent of all parameters.

Now following the steps of the proof of $(*)$ using (C.2) instead of (C.1) we can prove that for $p \geq 2$

(**) $\quad \operatorname{ess} \sup _{R_{+}^{\prime}} u_{+}^{p} \leq$

$C^{\frac{h}{h-1}}\left(\alpha^{2} \beta^{-1} \lambda(Q)^{-1}+1\right)^{1 /(h-1)}\left(\alpha^{-2} \beta \Lambda(Q)+1\right)^{h /(h-1)} \iint_{R} u_{+}^{p}\left(\alpha^{-2} \beta w_{2}+v\right) d x d t$

and Theorem B will follow from $\left({ }^{* *}\right)$ and an iteration argument like the one given in Lemma 3.4 of [GW2]. Finally we conclude Section 3 by making some comments about the proof of mean value inequalities for $u^{p}$, when $p<0$, where $u$ is a positive solution of (1.1). These inequalities will be necessary in the proof of Theorem A and in order to show them we need the following generalization of (C.2): 
(C.3) Let $-\infty<p<+\infty, p \neq 0,1$, $u$ satisfy $0<m<u(x, t)<M<\infty$ in $R, w_{2} \in A_{2}$. Then if $p>1$ and $u$ is a subsolution in $R$, or if $p<0$ and $u$ is a supersolution in $R$,

$\operatorname{ess} \sup _{\tau \in\left(t_{0}-\beta^{\prime}, t_{0}+\beta\right)} \int_{Q^{\prime}} u(x, \tau)^{p} v(x) d x+\frac{p-1}{p} \iint_{R_{+}^{\prime}}\left|\nabla_{\lambda} u^{p / 2}\right|^{2} w_{1}(x) d x d t$ $\leq c \iint_{R} u^{p}\left(\frac{p}{p-1} \cdot \frac{w_{2}(x)}{\left(\alpha-\alpha^{\prime}\right)^{2}}+\frac{v(x)}{\beta-\beta^{\prime}}\right) d x d t$.

Moreover, if $0<p<1$ and $u$ is a supersolution in $R$, then

$\operatorname{ess} \sup _{\tau \in\left(\mathrm{t}_{0}-\beta, t_{0}+\beta^{\prime}\right)} \int_{Q^{\prime}} u(x, \tau)^{p} v(x) d x+\left|\frac{p-1}{p}\right| \iint_{R^{\prime}}\left|\nabla_{\lambda} u^{p / 2}\right|^{2} w_{1} d x d t$ $\leq c \iint_{R} u^{p}\left(\left|\frac{p}{p-1}\right| \frac{w_{2}}{\left(\alpha-\alpha^{\prime}\right)^{2}}+\frac{v}{\beta-\beta^{\prime}}\right) d x d t$.

In this paper we do not present the proofs of (C.2) and (C.3) since their proofs are similar to the ones given in Section 2 of [GW2].

In Section 4, we prove

Theorem E: Let $v$ and $w_{1}$ be weights such that there exists $s>1$ with

(1.18) $\left(\frac{r(I)}{r(B)}\right)^{2}\left(\frac{1}{|I|} \int_{I}\left(\frac{v}{v(B)}\right)^{s} d x\right)^{1 / s}\left(\frac{1}{|I|} \int_{I}\left(\frac{w_{1}}{w_{1}(B)}\right)^{-s} d x\right)^{1 / s} \leq c$

for all $\delta$-balls $I, B$ with $I \subset 2 a^{2} B$ (a as in (1.9)), where $c$ is a constant independent of the balls. Let $Q=Q(\xi, r)$ and $\varphi$ be a $C^{1}$ function such that $\varphi \equiv 1$ in $Q(\xi, k r), 1 / 2 \leq k<1,0 \leq \varphi \leq 1$, supp $\varphi \subset Q$ and

$$
\varphi(x) \varphi\left(H\left(t_{0}, x, y\right)\right) \leq \varphi(H(t, x, y))
$$

for all $x, y, t, t_{0}$ with $0 \leq t \leq t_{0}$. Then, if $u \in \operatorname{Lip}(Q)$,

$\int_{Q}\left|u(x)-A_{Q}\right|^{2} \varphi(x) v(x) d x \leq c \frac{v(Q)}{w_{1}(Q)} r(Q)^{2} \int_{Q}\left|\nabla_{\lambda} u(x)\right|^{2} \varphi(x) w_{1}(x) d x$,

where $A_{Q}=\frac{1}{\varphi(Q)} \int_{Q} u(x) \varphi(x) d x$. 
Finally, in Section 5, we prove Theorem A. This theorem follows as an application of Bombieri's lemma ([GW2]). In order to verify the hypotheses of Bombieri's lemma we need Theorem B and Theorem F, which we state next. We write

$$
(v \otimes 1)(A)=\iint_{A} v(x) d x d t
$$

where $v=v(x), x \in \mathbf{R}^{n}$, and $A \subset \mathbf{R}^{n+1}=\left\{(x, t): x \in \mathbf{R}^{n}, t \in R\right\}$.

Theorem F: Suppose $v$ is a doubling weight, $w_{2} \in A_{2}$, (1.18) holds and $w_{2} v^{-1} \in A_{\infty}(v)$. Let $Q_{R}$ be a $\delta$-ball of radius $R, t_{0} \in(a, b)$ and $\tilde{w}_{2}=w_{2} / w_{2}\left(Q_{R}\right)$ and $\tilde{v}=v / v\left(Q_{R}\right)$. If $u$ is a solution of $(1.1)$ in $Q_{3 R / 2} \times(a, b)$ which is bounded below by a positive constant, then there are constants $c_{1}$, $M_{2}, x$ and $V$ such that if for $s>0$ we define

$$
\begin{aligned}
& E^{+}=\left\{(x, t) \in Q_{R} \times\left(t_{0}, b\right): \log u<-s-M_{2}\left(b-t_{0}\right)-V\right\} \\
& E^{-}=\left\{(x, t) \in Q_{R} \times\left(a, t_{0}\right): \log u>s-M_{2}\left(a-t_{0}\right)-V\right\},
\end{aligned}
$$

then

$$
\left(\left(\tilde{v}+\tilde{w}_{2}\right) \otimes 1\right)\left(E^{+}\right) \leq c_{1}\left(\frac{1}{s} \frac{v\left(Q_{R}\right)}{w_{1}\left(Q_{R}\right)} \frac{R^{2}}{b-t_{0}}\right)^{n}\left(b-t_{0}\right)
$$

and

$$
\left(\left(\tilde{v}+\tilde{w}_{2}\right) \otimes 1\right)\left(E^{-}\right) \leq c_{1}\left(\frac{1}{s} \frac{V\left(Q_{R}\right)}{w_{1}\left(Q_{R}\right)}-\frac{R^{2}}{t_{0}-a}\right)^{x}\left(t_{0}-a\right) .
$$

Here $c_{1}$ and $\varkappa$ depend only on the constants in the conditions on $v$ and $w_{2}$, $M_{2} \approx \frac{w_{2}\left(Q_{R}\right)}{R^{2} v\left(Q_{R}\right)}$, and $V$ is a constant which depends on $u$.

In order to prove this theorem, if we follow the steps of Lemma 4.9 of [GW2], we just have to verify that a certain test function (see [FL1]) satisfies the conditions of Theorem E. This will be done in Lemma 5.4.

\section{Interpolation Inequality}

In this section we prove Theorem $\mathrm{D}$. We start with

Theorem 2.1. Let $w_{1}, w_{2}$, and $\mu$ be doubling weights and suppose (1.17) holds for $w_{1}, w_{2}$ with any $\mu$, and for some $q>2$. If $Q=Q(\xi, r)$ and $w_{2} v^{-1} \in A_{\infty}(v)$ then there exist $h>1$ and a constant $c>0$, independent of $Q$ and $u$, such that 


$$
\begin{aligned}
& \frac{1}{w_{2}(Q)} \int_{Q}|u|^{2 h} w_{2} d x \\
& \leq c\left(\frac{1}{v(Q)} \int_{Q} u^{2} v d x\right)^{h-1}\left(\frac{r^{2}}{w_{1}(Q)} \int_{Q\left(\xi, a^{2} r\right)}\left|\nabla_{\lambda} u\right|^{2} w_{1} d x+\left(a v_{\mu, Q}|u|\right)^{2}\right)
\end{aligned}
$$

for all $u \in \tilde{H}\left(a^{2} Q\right)$, and $a$ as in (1.9). Also if (1.17) is replaced by (1.16), then

$$
\frac{1}{w_{2}(Q)} \int_{Q}|u|^{2 h} w_{2} d x \leq c\left(\begin{array}{c}
1 \\
v(Q)
\end{array} \int_{Q} u^{2} v d x\right)^{h-1}\left(\frac{r^{2}}{w_{1}(Q)} \int_{Q}\left|\nabla_{\lambda} u\right|^{2} w_{1} d x\right)
$$

for all $u \in \tilde{H}_{0}(Q)$.

PROOF: The proof follows as in [GW1], Theorem 3; the only differences are that we obtain $Q\left(\xi, a^{2} r\right)$ in the second integral on the right when we apply Poincaré's inequality and in the end we use the results of Calderón for weights in homogeneous spaces (see $[\mathrm{C}]$ ).

Corollary 2.2. Let $w_{1}, w_{2}$ be doubling weights and suppose (1.17) holds with $w_{1}, w_{2}, \mu=1$ and some $q>2$. If $w_{2} v^{-1} \in A_{\infty}(v)$, then there exists $h>1$ and a constant $c>0$ such that

$$
\begin{aligned}
& \frac{1}{w_{2}(Q)} \int_{Q}|u|^{2 h} w_{2} d x \\
& \leq c\left(\frac{1}{v(Q)} \int_{Q} u^{2} v d x\right)^{h-1}\left(\frac{r^{2}}{w_{1}(Q)} \int_{a^{2} Q}\left|\nabla_{\lambda} u\right|^{2} w_{1} d x+\frac{1}{v(Q)} \int_{Q} u^{2} v d x\right)
\end{aligned}
$$

for all $u \in \tilde{H}\left(a^{2} Q\right), Q=Q(\xi, r)$.

Proof: The conclusion of Theorem 2.1 holds for $\mu=1$. But, by Schwarz's inequality,

$$
\begin{aligned}
a v_{Q}|u| & =\frac{1}{|Q|} \int_{Q}|u| d x \\
& =\frac{1}{|Q|} \int_{Q} u v^{1 / 2} v^{-1 / 2} d x \leq \frac{1}{|Q|} \cdot\left(\int_{Q} u^{2} v d x\right)^{1 / 2}\left(\int_{Q} \frac{1}{v} d x\right)^{1 / 2} \\
& \leq\left(\frac{1}{v(Q)} \int_{Q} u^{2} v d x\right)^{1 / 2},
\end{aligned}
$$


where in the last inequality we used the fact that $v \in A_{2}$.

In the next section we prove mean value inequalities. In order to be able to iterate a certain inequality as was done in $[\mathrm{GW} 2]$ we need a refinement of the above corollary. This refinement is Theorem $\mathrm{D}$ and to prove it we need the following lemmas.

Lemma 2.3. Given $Q=Q(\xi, s)$ and $0<r<s$, there exists $x_{1}, \ldots, x_{m(r, s)}$ in $Q$, and $k \geq 1$ independent of $\xi, r, s$, such that

$$
Q\left(x_{j}, r / k\right) \bigcap Q\left(x_{h}, r / k\right)=\varnothing, h \neq j
$$

$$
Q(\xi, s) \subset \cup_{j=1}^{m(r, s)} Q\left(x_{j}, r\right) .
$$

Moreover, $m(r, s) \leq c\left(\frac{s}{r}\right)^{\nu^{\prime}}$ for some constant $\nu^{\prime}$ depending only on the dimension.

ProOF: If we apply Theorem 1.2, page 69 , of $[\mathrm{CoW}]$ to the open covering of $Q$ given by $\left(S(x, r / 4 a)_{x \in Q}\right.$, there exist $x_{1}, \ldots, x_{m(r, s)}$ in $Q$ such that: $S\left(x_{h}, r / 4 a\right) \bigcap S\left(x_{j}, r / 4 a\right)=\varnothing$ if $j \neq h$ and $Q(\xi, s) \subset \cup_{j=1}^{m(r)} S\left(x_{j}, r / a\right)$. By (1.9), $S\left(x_{j}, r / 4 a\right) \supset Q\left(x_{j}, r / 4 a^{2}\right)$ and $S\left(x_{j}, r / a\right) \subset Q\left(x_{j}, r\right)$. Therefore, if we choose $k=4 a^{2}$, (i) and (ii) follow. It remains to find an upper bound for $m(r, s)$. First, we note that $Q\left(x_{j}, r / k\right) \subset Q\left(\xi, a^{2}(k+1) s / k\right)$. But

$$
\frac{r}{k}=\frac{2 a^{4}(k+1) s}{k} \frac{r}{2 a^{4}(k+1) s},
$$

and so by (1.10), there exists $\nu^{\prime}>0$, such that

$$
\left|Q\left(x_{j}, \frac{r}{k}\right)\right| \geq\left(\frac{r}{2 a^{4}(k+1) s}\right)^{\nu^{\prime}}\left|Q\left(x_{j}, \frac{2 a^{4}(k+1) s}{k}\right)\right|
$$

and since the $Q\left(x_{j}, r / k\right)$ are disjoint, 


$$
\begin{aligned}
\left|Q\left(\xi, \frac{a^{2}(k+1) s}{k}\right)\right| & \geq \sum_{j}\left|Q\left(x_{j}, \frac{r}{k}\right)\right| \\
& \geq c\left(\frac{r}{s}\right)^{\nu} \sum_{j}\left|Q\left(x_{j}, \frac{2 a^{4}(k+1) s}{k}\right)\right|
\end{aligned}
$$

But,

$$
Q\left(x_{j}, \frac{2 a^{4}(k+1) s}{k}\right) \supset Q\left(\xi, \frac{a^{2}(k+1) s}{k}\right)
$$

and so

$$
\left|Q\left(\xi, \frac{a^{2}(k+1) s}{k}\right)\right| \geq c\left(\frac{r}{s}\right)^{\nu} m(r, s)\left|Q\left(\xi, \frac{a^{2}(k+1) s}{k}\right)\right| .
$$

Therefore, $m(r, s) \leq c(s / r)^{\nu}$.

Lemma 2.4. If $\delta(y, z)<s$ then $F_{j}\left(z^{*}, s\right) \leq\left(2 a^{2}\right)^{G_{j}} F_{j}\left(y^{*}, s\right), G_{j}$ as in (1.10).

PROOF: Since $Q(z, s) \subset Q\left(y, 2 a^{2} s\right), F_{j}\left(z^{*}, s\right) \leq F_{j}\left(y^{*}, 2 a^{2} s\right)$. By (1.10), it follows that

$$
F_{j}\left(z^{*}, s\right) \leq F_{j}\left(y^{*}, 2 a^{2} s\right) \leq\left(2 a^{2}\right)^{G_{j}} F_{j}\left(y^{*}, s\right) .
$$

Lemma 2.5. If $0<\epsilon<1$ and $\eta \in Q=Q\left(\xi\right.$, s), then $Q\left(\eta, \epsilon S /\left(2 a^{2}\right)^{\zeta}\right) \subset$ $Q(\xi,(1+\epsilon) s)$, where $\zeta=\max _{j=1, \ldots, n} G_{j}$.

Proof: If $y \in Q\left(\eta, \epsilon S /\left(2 a^{2}\right)^{\zeta}\right)$ then by (1.8), $\left|y_{j}-\eta_{j}\right| \leq F_{j}\left(\eta^{*}, \epsilon S /\left(2 a^{2}\right)^{\zeta}\right)$ and by (1.10) and Lemma 2.4

$$
F_{j}\left(\eta^{*}, \frac{\epsilon s}{\left(2 a^{2}\right)^{\zeta}}\right) \leq \frac{\epsilon}{\left(2 a^{2}\right)^{\zeta}} F_{j}\left(\eta^{*}, s\right) \leq \epsilon F_{j}\left(\xi^{*}, s\right) .
$$

Therefore

$$
\begin{aligned}
\left|y_{j}-\xi_{j}\right| & \leq\left|y_{j}-\eta_{j}\right|+\left|\eta_{j}-\xi_{j}\right| \leq \epsilon F_{j}\left(\xi^{*}, s\right)+F_{j}\left(\xi^{*}, s\right) \\
& =(1+\epsilon) F_{j}\left(\xi^{*}, s\right) \\
& \leq F_{j}\left(\xi^{*},(1+\epsilon) s\right),
\end{aligned}
$$

where in the last inequality we used (1.10).

\section{Proof of Theorem D.}

Let $Q=Q(\xi, s)$. By Lemma $2.5, \delta(Q, \partial(1+\epsilon) Q) \geq \epsilon S /\left(2 a^{2}\right)^{5}$. Apply Lemma 2.3 to $r=\frac{\epsilon S}{\left(2 a^{2}\right)^{\zeta} a^{2}}$ to find $x_{1}, \ldots, x_{m(r, s)} \in Q$ such that: $Q\left(x_{j}, r / k\right) \bigcap Q\left(x_{h}, r / k\right)=\varnothing$ if $j \neq h, Q(\xi, s) \subset \cup_{j=1}^{m(r, s)} Q\left(x_{j}, r\right)$ and $m(r, s) \leq c(s / r)^{\nu}$. 
Note that, by (2.5), $Q\left(x_{j}, a^{2} r\right)=Q\left(x_{j}, \frac{\epsilon S}{\left(2 a^{2}\right)^{\zeta}}\right) \subset Q(\xi,(1+\epsilon) s)=(1+\epsilon) Q$.

Then using Corollary 2.2, doubling for $w_{2}$, doubling for $v$ and $w_{1}$ and the fact that $Q\left(x_{j}, 2 a^{2} s\right) \supset Q(\xi, s)$ and $Q\left(\xi, 2 a^{2} s\right) \supset Q\left(x_{j}, s\right)$,

$$
\begin{aligned}
& \int_{Q}|u|^{2 h} w_{2} d x \leq \sum_{j=1}^{m(r, s)} \int_{Q\left(x_{j}, r\right)}|u|^{2 h} w_{2} d x \\
& \leq c \sum_{j=1}^{m(r, s)} w_{2}\left(Q\left(x_{j}, r\right)\right)\left(\frac{1}{v\left(Q\left(x_{j}, r\right)\right.} \int_{Q\left(x_{j}, r\right)} u^{2} v d x\right)^{h-1} \\
& \cdot\left\{\frac{r^{2}}{w_{1}\left(Q\left(x_{j}, r\right)\right)} \int_{Q\left(x_{j}, a^{2} r\right)}\left|\nabla_{\lambda} u\right|^{2} w_{1} d x+\frac{1}{v\left(Q\left(x_{j}, r\right)\right)} \int_{Q\left(x_{j}, r\right)} u^{2} v d x\right\} \\
& \leq c\left(\frac{s}{r}\right)^{\nu^{\prime}} w_{2}(Q(\xi, s))\left[\left(\frac{r}{2 a^{2} s}\right)^{-\alpha} \frac{1}{v(Q(\xi, s))} \int_{(1+c) Q} u^{2} v d x\right]^{h-1} \\
& \cdot\left\{\frac{s^{2}}{w_{1}(Q(\xi, s))}-\left(\frac{r}{2 a^{2} s}\right)^{-\alpha} \int_{(1+\epsilon) Q}\left|\nabla_{\lambda} u\right|^{2} w_{1} d x\right. \\
&+\left.\left(\frac{r}{2 a^{2} s}\right)^{-\alpha} \frac{1}{v(Q(\xi, s))} \int_{(1+\epsilon) Q} u^{2} v d x\right\} .
\end{aligned}
$$

The theorem follows if we choose $b=\nu+2 \alpha$, since $s / r=c \epsilon^{-1}$.

\section{Mean value inequalities.}

In this section we prove Theorem B and some other mean value inequalities. Since the proofs are similar to the ones given by [GW2], we just point out the differences. Basically, we have to be a little more careful in the iteration argument since there is a factor $\epsilon$ in Theorem D.

We asume throughout this section that:

(a) $w_{1}, w_{2}, v \in A_{2}$

(b) Poincaré's inequality, (1.17), holds for both of the pairs $w_{1}$, $w_{2}$ and $w_{1}, v$ with some $q>2$ and $\mu=1$

(c) $w_{2} v^{-1} \in A_{\infty}(v)$.

Denote $R_{r, s}=Q\left(x_{0}, r\right) \times\left(t_{0}-s, t_{0}+s\right)$ and let $R=R_{r, s}, R^{\prime}=R_{Q, \sigma}$ with $r / 2<\varrho<r$ and $s / 2<\sigma<s$ and define 


$$
C=c \frac{r^{2+b} s}{(r-\varrho)^{2+b}(s-\sigma)}
$$

where $b$ is given by Theorem $\mathrm{D}$ and $c$ is a constant that may vary, but which only depends on the weights and on $h$, where $h>1$ is the index for which Theorem $\mathrm{D}$ holds for both $w_{2}$ and $v$ on the left hand side.

We also write $\lambda(Q)=w_{1}(Q) / v(Q)$ and $\Lambda(Q)=w_{2}(Q) / v(Q)$. We start this section with the proof of (C.1). This estimate will be important in deducing a mean value inequality for subsolutions of (1.1).

Proof OF (C.1): If $u \in H$ define

$$
\varphi(x, t)=\eta^{2}(x, t)\left[\int_{0}^{u(x, t)} H_{M}^{\prime}(s)^{2} d s+u(x, t) H_{M}^{\prime}(u(x, t))^{2}\right] \chi\left(t, \tau_{1}, \tau_{2}\right),
$$

where $\eta \in C_{0}^{\infty}(R)$ will be specified later, $t_{0}-s<\tau_{1}<\tau_{2}<t_{0}+s$ and $\chi\left(t, \tau_{1}, \tau_{2}\right)$ denotes the characteristic function of $\left(\tau_{1}, \tau_{2}\right)$. The fact that the function $\varphi$ is in $H_{0}$ follows as a consequence of the following result: if $f$ is a piecewise smooth function on the real line with $f^{\prime} \in L^{\infty}(-\infty, \infty)$ and if $u \in H$, then $f \circ u \in H$. Here we use the convention that $f^{\prime}(u)=0$ if $u \in L$ where $L$ denotes the set of corner points of $f$ (the proof follows the steps of Theorem 7.8 of $[\mathrm{GT}]$ and it also shows that $\nabla_{\lambda}(f \circ u)=f^{\prime}(u) \nabla_{\lambda} u$ and $\left.(f(u))_{t}=f^{\prime}(u) u_{t}\right)$. The proof of the above fact also verifies that in our case $\varphi \geq 0$ in the $H_{0}$-sense since $H_{M}(s)=0$ for $s<0$.

Since $u$ is a subsolution, we have

$$
\iint_{R}\left(\langle A \nabla u, \nabla \varphi\rangle+u_{t} \varphi v\right) d x d t \leq 0
$$

Note that by another limiting argument

$u_{t}\left[\eta^{2} \int_{0}^{u} H_{M}^{\prime}(s)^{2} d s\right]=\left[u \eta^{2} \int_{0}^{u} H_{M}^{\prime}(s)^{2} d s\right]_{t}-u\left(\eta^{2}\right)_{t} \int_{0}^{u} H_{M}^{\prime}(s)^{2} d s-\eta^{2} H_{M}^{\prime}(u)^{2} u_{t} u$,

and then by definition of $\varphi$, for $\tau_{1}<t<\tau_{2}$,

$$
u_{t} \varphi=\left[u \eta^{2} \int_{0}^{u} H_{M}^{\prime}(s)^{2} d s\right]_{t}-\left(\eta^{2}\right)_{t} u \int_{0}^{u} H_{M}^{\prime}(s)^{2} d s
$$

and 
$\nabla \varphi=2 \eta \nabla \eta\left[\int_{0}^{u} H_{M}^{\prime}(s)^{2} d s+u H_{M}^{\prime}(u)^{2}\right]+\eta^{2}\left[H_{M}^{\prime}(u)^{2} \nabla u+f_{M}^{\prime}(u) \nabla u\right]$,

where $f_{M}(s)=s H_{M}^{\prime}(s)^{2}$ (note that $\nabla\left(f_{M}(u)\right)=f_{M}^{\prime}(u) \nabla u$, since $f_{M}$ is piecewise smooth with $f_{M}^{\prime} \in L^{\infty}$ ). If we substitute the two last equations in (3.3) we get, with $Q=Q\left(x_{0}, r\right)$,

$$
\begin{aligned}
& \int_{Q} \int_{\tau_{1}}^{\tau_{2}}\left[u \eta^{2} \int_{0}^{u} H_{M}^{\prime}(s)^{2} d s\right]_{t} v d x d t+\int_{Q} \int_{\tau_{1}}^{\tau_{2}} \eta^{2} H_{M}^{\prime}(u)^{2}\langle A \nabla u, \nabla u\rangle d x d t \\
\leq & \int_{Q} \int_{\tau_{1}}^{\tau_{2}}\left[\left(\eta^{2}\right)_{t} u \int_{O}^{u} H_{M}^{\prime}(s)^{2} d s\right] v d x d t \\
- & 2 \int_{Q} \int_{\tau_{1}}^{\tau_{2}} \eta\langle A \nabla u, \nabla \eta\rangle\left[\int_{0}^{u} H_{M}^{\prime}(s)^{2} d s+u H_{M}^{\prime}(u)^{2}\right] d x d t \\
- & \int_{Q} \int_{\tau_{1}}^{\tau_{2}} \eta^{2}\langle A \nabla u, \nabla u\rangle f_{M}^{\prime}(u) d x d t .
\end{aligned}
$$

We can drop the last term on the right since the integrand is non-negative. The second term on the right is majorized in absolute value by

$$
\begin{aligned}
& 4 \int_{Q} \int_{\tau_{1}}^{\tau_{2}}|\langle A \nabla u, \nabla \eta\rangle| \eta H_{M}^{\prime}(u)^{2} u d x d t \\
& \quad=4 \int_{Q} \int_{\tau_{1}}^{\tau_{2}}\left|\left\langle A H_{M}^{\prime}(u) \eta \nabla u, u H_{M}^{\prime}(u) \nabla \eta\right\rangle\right| d x d t \\
& \quad \leq 2 \epsilon \int_{Q} \int_{\tau_{1}}^{\tau_{2}}\left\langle A \nabla\left(H_{M}(u)\right), \nabla\left(H_{M}(u)\right)\right\rangle, \eta^{2} d x d t \\
& \quad+\frac{2}{\epsilon} \int_{Q} \int_{\tau_{1}}^{\tau_{2}}\langle A \nabla \eta, \nabla \eta\rangle u^{2} H_{M}^{\prime}(u)^{2} d x d t
\end{aligned}
$$

where we used the fact that $|\langle A x, y\rangle| \leq\langle A x, x\rangle^{1 / 2}\langle A y, y\rangle^{1 / 2} \leq \frac{\epsilon}{2}\langle A x, x\rangle+$ $\frac{1}{2 \epsilon}\langle A y, y\rangle$. If we pick $\epsilon=\frac{1}{4}$ - we get 


$$
\begin{aligned}
& \int_{Q} \int_{\tau_{1}}^{\tau_{2}}\left[u \eta^{2} \int_{0}^{u} H_{M}^{\prime}(s)^{2} d s\right]_{t} v d x d t \\
& +\frac{1}{2} \int_{Q} \int_{\tau_{1}}^{\tau_{2}} \eta^{2}\left\langle A \nabla\left(H_{M}(u)\right), \nabla\left(H_{M}(u)\right)\right\rangle d x d t \\
& \leq 8 \int_{Q} \int_{\tau_{1}}^{\tau_{2}}\langle A \nabla \eta, \nabla \eta\rangle u^{2} H_{M}^{\prime}(u)^{2} d x d t+\int_{Q} \int_{\tau_{1}}^{\tau_{2}}\left[\left(\eta^{2}\right)_{t} u \int_{0}^{u} H_{M}^{\prime}(s)^{2} d s\right] v d x d t .
\end{aligned}
$$

Choosen $\eta$ to be zero in a neighborhood of $\left\{\partial Q \times\left(t_{0}-s, t_{0}+s\right)\right\} \cup\{Q \times(t=$ $\left.\left.t_{0}-s\right)\right\}, \eta \equiv 1$ in $R_{+}^{\prime}, 0 \leq \eta \leq 1,\left|\nabla_{\lambda} \eta\right| \leq c /(r-\varrho),\left|\eta_{t}\right| \leq c /(s-\sigma)$ (see page 537 of [FL1]). If we pick $\tau_{1}$ so close to $t_{0}-s$ that $\eta\left(x, \tau_{1}\right)=0$ for all $x \in Q$, drop the second term on the left of (3.4) (which is non-negative) and use Lemma 5 of $[\mathrm{AS}]$ it follows that

$$
\begin{aligned}
& \text { (3.5) } \operatorname{ess} \sup _{\tau_{2} \in\left(t_{0}-\sigma, t_{0}+s\right)} \int_{Q^{\prime}} u\left(x, \tau_{2}\right) \int_{0}^{u\left(x, \tau_{2}\right)} H_{M}^{\prime}(s)^{2} d s v d x \\
& \quad \leq c \iint_{R} u^{2} H_{M}^{\prime}(u)^{2}\left[\frac{w_{2}}{(r-\varrho)^{2}}+\frac{v}{s-\sigma}\right] d x d t .
\end{aligned}
$$

If we fix $\tau_{2} \in\left(t_{0}-\sigma, t_{0}+s\right)$ and $\tau_{1}$ as before and if we drop the first term on the left of (3.4) (which we can see is non-negative after performing the integration) we obtain

$$
\begin{aligned}
& \int_{Q} \int_{\tau_{1}}^{\tau_{2}} \eta^{2}\left\langle A \nabla\left(H_{M}(u)\right),\right.\left.\nabla\left(H_{M}(u)\right)\right\rangle d x d t \\
& \leq c \iint_{R} u^{2} H_{M}^{\prime}(u)^{2}\left[\frac{w_{2}}{(r-\varrho)^{2}}+\frac{v}{s-\sigma}\right] d x d t
\end{aligned}
$$

Letting $\tau_{2} \rightarrow t_{0}+s$ and using (1.2) we get

$$
\iint_{R_{+}^{\prime}}\left|\nabla_{\lambda}\left(H_{M}(u)\right)\right|^{2} w_{1} d x d t \leq c \iint_{R} u^{2} H_{M}^{\prime}(u)^{2}\left[\frac{w_{2}}{(r-\varrho)^{2}}+\frac{v}{s-\sigma}\right] d x d t
$$

Finally note that 
Mean Value and Harnack Inequalities

265

$$
\begin{aligned}
H_{M}(u)^{2}= & \int_{0}^{u}\left(H_{M}(s)^{2}\right)^{\prime} d s=\int_{0}^{u} 2 H_{M}(s) H_{M}^{\prime}(s) d s \\
& \leq 2 \int_{0}^{u} s H_{M}^{\prime}(s)^{2} d s \leq 2 u \int_{0}^{u} H_{M}^{\prime}(s)^{2} d s,
\end{aligned}
$$

since $H_{M}(s) \leq s H_{M}^{\prime}(s)$. Combining this with (3.5) and (3.7), (C.1) follows with $\alpha, \beta, \alpha^{\prime}, \beta^{\prime}$ taken there to be $r, s, \varrho, \sigma$.

Lemma 3.8. Let $p \geq 2, R, R^{\prime}$ be as defined above and assume (3.1) holds. If $u$ is a subsolution of (1.1) in $R$, then $u_{+}$is bounded in $R_{+}^{\prime}=$ $Q\left(x_{0}, \varrho\right) \times\left(t_{0}-\sigma, t_{0}+s\right)$ and

iss $\sup _{R_{+}^{\prime}} u_{+}^{p}$

$$
\leq\left(p^{2} C\right)^{\frac{h}{h-1}}\left(1+\frac{r^{2}}{s} \frac{1}{\lambda(Q)}\right)^{\frac{1}{h-1}}\left(1+\frac{s}{r^{2}} \Lambda(Q)\right)^{\frac{h}{h-1}} \iint_{R} u_{+}^{p}\left(\frac{s}{r^{2}} w_{2}+v\right) d x d t,
$$

with $C$ as in (3.2).

Proof: $\quad H_{M}(u)$ is a function in $H$ since $u \in H$ and $H_{M}$ is a $C^{1}$ function with bounded derivative. Then by Fubini's theorem we have that $H_{M}(u(., \tau)) \in \tilde{H}$ for ace. $\tau \in\left(t_{0}-\sigma, t_{0}+s\right)$. If we apply Theorem $\mathrm{D}$ to the function $F(x)=$ $H_{M}(u(x, \tau)), Q=Q_{\varrho}$ and $\epsilon>0$ such that $(1+\epsilon) \varrho<r$ and combine this with (C.1) we obtain

$$
\begin{aligned}
& \frac{1}{w_{2}\left(Q_{\varrho}\right)} \int_{Q_{\varrho}} H_{M}\left(u(x, \tau)^{2 h} w_{2}(x) d x\right. \\
\leq & c \epsilon^{-b}\left\{\frac{1}{v\left(Q_{\varrho}\right)} \iint_{R} u^{2} H_{M}^{\prime}(u)^{2}\left(\frac{w_{2}}{(r-(1+\epsilon) \varrho)^{2}}+\frac{v}{s-\sigma}\right) d x d t\right\}^{h-1} \\
& \cdot\left\{\frac{\varrho^{2}}{w_{1}\left(Q_{\varrho}\right)} \int_{Q_{(1+\epsilon) e}}\left|\nabla_{\lambda}\left(H_{M}(u(x, \tau))\right)\right|^{2} w_{1}(x) d x\right. \\
+ & \left.\frac{1}{v\left(Q_{\varrho}\right)} \iint_{R} u^{2} H_{M}^{\prime}(u)^{2}\left(\frac{w_{2}}{(r-(1+\epsilon) \varrho)^{2}}+\frac{v}{s-\sigma}\right) d x d t\right\}
\end{aligned}
$$

for a.e. $\tau \in\left(t_{0}-\sigma, t_{0}+s\right)$. 
266

José C. Fernandes

Integrate with respect to $\tau$ over $\left(t_{0}-\sigma, t_{0}+s\right)$ and apply (C.1) to get

$$
\begin{aligned}
& \frac{1}{w_{2}\left(Q_{\varrho}\right)} \iint_{R_{+}^{\prime}} H_{M}(u(x, t))^{2 h} w_{2}(x) d x d t \\
& \left.\leq c \frac{\epsilon^{-b}}{v\left(Q_{\varrho}\right)^{h-1}}\left(\frac{\varrho^{2}}{w_{1}\left(Q_{\varrho}\right)}+\frac{s+\sigma}{v\left(Q_{\varrho}\right)}\right)\left(\iint_{R} u^{2} H_{M}^{\prime}(u)^{2} \frac{w_{2}}{(r-(1+\epsilon) \varrho)^{2}}+\frac{v}{s-\sigma}\right) d x d t\right)^{h}
\end{aligned}
$$

Since $(r / 2)<\varrho<r$ and $(s / 2)<\sigma<s$, by the doubling property of the weights and the definitions of $\lambda$ and $\Lambda$, it follows that

$$
\begin{aligned}
& \frac{1}{\mathrm{w}_{2}\left(Q_{r}\right)} \iint_{R_{+}^{\prime}} H_{M}(u(x, t))^{2 h} w_{2}(x) d x d t \\
& \leq c \frac{\epsilon^{-b}}{v\left(Q_{r}\right)^{h}}\left(\frac{r^{2}}{\lambda\left(Q_{r}\right)}+s\right)\left(\iint_{R} u^{2} H_{M}^{\prime}(u)^{2}\left(\frac{w_{2}}{(r-(1+\epsilon) \varrho)^{2}}+\frac{v}{s-\sigma}\right) d x d t\right)^{h} .
\end{aligned}
$$

A similar inequality holds with $w_{2}$ replaced by $v$ on the left, and if we add the two inequalities, we obtain

$$
\begin{aligned}
& \text { (3.9) } \iint_{R_{+}^{\prime}} H_{M}(u)^{2 h}\left(\frac{w_{2}}{w_{2}\left(Q_{r}\right)}+\frac{v}{v\left(Q_{r}\right)}\right) d x d t \\
& \leq c \frac{\epsilon^{-b}}{v\left(Q_{r}\right)^{h}}\left(\frac{r^{2}}{\lambda\left(Q_{r}\right)}+s\right)\left(\iint_{R} u^{2} H_{M}^{\prime}(u)^{2}\left(\frac{w_{2}}{(r-(1+\epsilon) \varrho)^{2}}+\frac{v}{s-\sigma}\right) d x d t\right)^{h}
\end{aligned}
$$

for any $\epsilon$ such that $(1+\epsilon) \varrho<r$.

Now note that

$$
\begin{gathered}
\frac{w_{2}}{(r-(1+\epsilon) \varrho)^{2}}+\frac{v}{s-\sigma} \leq \frac{r^{2}}{(r-(1+\epsilon) \varrho)^{2}(s-\sigma)}\left\{\frac{s}{r^{2}} w_{2}+v\right\}, \\
\iint_{R_{+}^{\prime}}\left\{\frac{w_{2}}{w_{2}\left(Q_{r}\right)}+\frac{v}{v\left(Q_{r}\right)}\right\} d x d t \approx s, \\
\iint_{R}\left\{\frac{s}{r^{2}} w_{2}+v\right\} d x d t \approx s\left\{\frac{s}{r^{2}} w_{2}\left(Q_{r}\right)+v\left(Q_{r}\right)\right\} \approx s v\left(Q_{r}\right)\left\{\frac{s}{r^{2}} \Lambda\left(Q_{r}\right)+1\right\}, \\
\frac{s r^{-2} w_{2}(x)+v(x)}{s r^{-2} w_{2}\left(Q_{r}\right)+v\left(Q_{r}\right)} \leq \frac{w_{2}(x)}{w_{2}\left(Q_{r}\right)}+\frac{v(x)}{v\left(Q_{r}\right)} .
\end{gathered}
$$


Thus, by raising both sides of (3.9) to the power $1 / h$, normalizing and using the fact that $\epsilon^{-b / h} \leq \epsilon^{-b}$, we obtain

$$
\begin{aligned}
& \left(\iint_{R_{+}^{\prime}} H_{M}(h)^{2 h}\left(\frac{s}{r^{2}} w_{2}+v\right) d x d t\right)^{1 / h} \\
& \leq c \epsilon^{-b} \frac{r^{2} s}{(r-(1+\epsilon) \varrho)^{2}}(s-\sigma) \\
& \quad \cdot \iint_{R} u^{2} H_{M}^{\prime}(u)^{2}\left(\frac{s}{r^{2}} w_{2}+v\right) d x d t
\end{aligned}
$$

for any $\epsilon$ such that $(1+\epsilon) \varrho<r$. Since $u_{+}^{p / 2} \chi_{\{0<u<M\}} \leq H_{M}(u)$ and $u H_{M}^{\prime}(u)$ $\leq p u_{+}^{p / 2} / 2$, if we let $M \rightarrow \infty$ it follows by Fatou's lemma that

$$
\begin{aligned}
& \left(\iint_{R_{+}^{\prime}} u_{+}^{p h}\left(\frac{s}{r^{2}} w_{2}+v\right) d x d t\right)^{1 / h} \\
& \leq c p^{2} \epsilon^{-b} \frac{r^{2} s}{(r-(1+\epsilon) \varrho)^{2}(s-\sigma)}\left(1+\frac{s}{r^{2}} \Lambda\left(Q_{r}\right)\right)\left(1+\frac{r^{2}}{s} \frac{1}{\lambda\left(\frac{\left.Q_{r}\right)}{)}\right.}\right)^{1 / h} \\
& \quad \cdot \iint_{R} u_{+}^{p}\left(\frac{s}{r^{2}} w_{2}+v\right) d x d t .
\end{aligned}
$$

Now, we have to iterate (3.11). Fix $r, s, \varrho, \sigma$ with $r / 2<\varrho<r$ and $s / 2<\sigma<s$. For $k=1,2, \ldots$ define sequences $\left\{s_{k}\right\}_{k \in \mathbf{N}}$ and $\left\{r_{k}\right\}_{k \in \mathbf{N}}$ and $\left\{\epsilon_{k}\right\}_{k \in \mathbf{N}}$ by $s_{1}=s, s_{k}-s_{k+1}=\frac{s-\sigma}{2^{k}}$ for $k \geq 1, r_{1}=r, r_{k}-r_{k+1}=(r-\varrho) / 2^{k}$ for $k \geq 1$, and $\epsilon_{k}=\frac{r-\varrho}{2^{k} r_{k}}=\frac{r_{k}-r_{k+1}}{r_{k}}$ for $k \geq 1$. Also, define $R_{k}=Q_{k} \times\left(t_{0}-s_{k}, t_{0}+s\right)$ for $k \geq 1$, where $Q_{k}=Q\left(x, r_{k}\right)$. Note that $R_{1}=R$ and $\bigcap_{k=1}^{\infty} R_{k}=R_{+}^{\prime}$. Since

$$
\frac{1}{2} s r^{-2} \leq s_{k} r_{k}^{-2} \leq 4 s r^{-2}
$$

if we apply (3.11) with $p$ replaced by $p h^{k-1}, p \geq 2$, and $r=r_{k}, \varrho=r_{k+1}$ and $\epsilon=\epsilon_{k+1}$ (note that $\left.\left(1+\epsilon_{k+1}\right) r_{k+1}<r_{k}\right)$, we obtain 
268 JosÉ C. FERNANDES

$$
\begin{aligned}
& \left(\iint_{R_{k+1}} u_{+}^{p h^{k}}\left(\frac{s}{r^{2}} w_{2}+v\right) d x d t\right)^{1 / h^{k}} \\
& \leq\left\{c\left(p h^{k-1}\right)^{2} \epsilon_{k+1}^{-b}-\frac{r_{k}^{2} s_{k}}{\left(r_{k}-\left(1+\epsilon_{k+1}\right) r_{k+1}\right)^{2}\left(s_{k}-s_{k+1}\right)}\left(1+\frac{s}{r^{2}} \Lambda\left(Q_{r}\right)\right)\right. \\
& \left.\quad\left(1+\frac{r^{2}}{s} \frac{1}{\lambda\left(Q_{r}\right)}\right)^{1 / h}\right\}^{1 /\left(h^{k-1}\right)} \cdot\left\{\iint_{R_{k}} u_{+}^{p h^{k-1}}\left(\frac{s}{r^{2}} w_{2}+v\right) d x d t\right\}^{1 /\left(h^{k-1}\right)}
\end{aligned}
$$

But note that

$$
\begin{aligned}
& \epsilon_{k+1}^{-b}-\frac{r_{k}^{2} s_{k}}{\left[r_{k}-\left(1+\epsilon_{k+1}\right) r_{k+1}\right]^{2}\left(s_{k}-s_{k+1}\right)} \\
& \quad=2^{(k+1) b} \frac{r_{k+1}^{b}}{(r-\varrho)^{b}} \frac{r_{k}^{2} s_{k}}{\left(\frac{r-\varrho}{2^{k}}-\frac{r-\varrho}{2^{k+1}}\right)^{2}\left(\frac{s-\sigma}{2^{k}}\right)} \\
& \quad \leq c 2^{(3+b) k} \frac{r^{2+b} s}{(r-\varrho)^{2+b}(s-\sigma)} \\
& \quad \leq C 2^{(3+b) k}
\end{aligned}
$$

where $C$ is given by (3.2). Thus,

$$
\begin{aligned}
& \left(\iint_{R_{k+1}} u_{+}^{p h^{k}}\left(\frac{s}{r^{2}} w_{2}+v\right) d x d t\right)^{1 / h^{k}} \\
& \leq\left\{C\left(p h^{k-1}\right)^{2} 2^{(3+b) k}\left(1+\frac{s}{r^{2}} \Lambda\left(Q_{r}\right)\right)\left(1+\frac{r^{2}}{s} \frac{1}{\lambda\left(Q_{r}\right)}\right)^{1 / h}\right\}^{1 / h^{k-1}} \\
& \cdot\left\{\iint_{R_{k}} u_{+}^{p h^{k-1}}\left(\frac{s}{r^{2}} w_{2}+v\right) d x d t\right\}^{1 / h^{k-1}} .
\end{aligned}
$$

If we iterate (3.12), we obtain 
ess $\sup _{R_{+}^{\prime}} u_{+}^{p}$

$$
\begin{aligned}
\leq \prod_{k=1}^{\infty}\left\{C\left(p h^{k-1}\right)^{2} 2^{(3+b) k}\left(1+\frac{s}{r^{2}} \Lambda\left(Q_{r}\right)\right)\right. \\
\left.\cdot\left(1+\frac{r^{2}}{s} \frac{1}{\lambda\left(Q_{r}\right)}\right)^{1 / h}\right\}^{1 / h^{k-1}} \iint_{R} u_{+}^{p}\left(\frac{s}{r^{2}} w_{2}+v\right) d x d t .
\end{aligned}
$$

Since $\Sigma_{k=1}^{\infty} \frac{1}{h^{k-1}}=\frac{h}{h-1}$ and $\Sigma_{k=1}^{\infty} \frac{k}{h^{k-1}}=\left(\frac{h}{h-1}\right)^{2}$, it follows that ess $\sup _{R_{+}^{\prime}} u_{+}^{p}$

$\leq\left(p^{2} C\right)^{\frac{h}{h-1}}\left(1+\frac{s}{r^{2}} \Lambda\left(Q_{r}\right)\right)^{\frac{h}{h-1}}\left(1+\frac{r^{2}}{s} \frac{1}{\lambda\left(Q_{r}\right)}\right)^{\frac{1}{h-1}} \iint_{R} u_{+}^{p}\left(\frac{s}{r^{2}} w_{2}+v\right) d x d t$

and this proves the lemma. Note that if we apply the above result for $p=2$, it follows that $u_{+}$is bounded on $R_{+}^{\prime}$.

ProOF OF THEOREM B: By Lemma 3.8 we know that $u_{+}$is bounded in $Q_{(1+\epsilon) e} \times\left(t_{0}-\sigma, t_{0}+s\right)$ for all $\epsilon$ such that $(1+\epsilon) \varrho<r$. If we define $F(x)=u_{+}^{p / 2}(x, \tau)$ then $F \in \tilde{H}\left(Q_{(1+\epsilon) e}\right)$ for a.e. $\tau \in\left(t_{0}-\sigma, t_{0}+s\right)$ and if we follow the proof of Lemma 3.8 using (C.2) instead of (C.1), we get (see the comments in the introduction)

ess $\sup _{R_{+}^{\prime}} u_{+}^{p}$

$\leq C^{\frac{h}{h-1}}\left(1+\frac{r^{2}}{s} \frac{1}{\lambda(Q)}\right)^{\frac{1}{h-1}}\left(1+\frac{s}{r^{2}} \Lambda(Q)\right)^{\frac{h}{h-1}} \iint_{R} u_{+}^{p}\left(\frac{s}{r^{2}} w_{2}+v\right) d x d t$

for $p \geq 2$. For $0<p<2$, define $I_{p}$ and $I_{\infty}$ as in Lemma 3.4 of [GW2]. The only difference in our case is that

$$
I_{\infty}\left(\alpha^{\prime}, \beta^{\prime}\right)^{2} \leq c\left[\frac{1}{\left(\alpha-\alpha^{\prime}\right)^{2+b}\left(\beta-\beta^{\prime}\right)}\right]^{\frac{h}{h-1}} I_{2}(\alpha, \beta)^{2}
$$


if $1 / 2<\alpha^{\prime}<\alpha<1$ and $1 / 2<\beta^{\prime}<\beta<1$. Thus, arguing as in Lemma 3.4 of [GW2] we prove that if $u$ is a solution of (1.1) and $p>0$ then

(3.13) $\quad \operatorname{ess} \sup _{R_{+}^{\prime}} u_{+}^{p} \leq$

$$
D\left(1+\frac{r^{2}}{s} \frac{1}{\lambda(Q)}\right)^{\frac{1}{h-1}}\left(1+\frac{s}{r^{2}} \Lambda(Q)\right)^{\frac{h}{h-1}} \iint_{R} u_{+}^{p}\left(\frac{s}{r^{2}} w_{2}+v\right) d x d t
$$

where $D$ is as in Theorem B.

If we apply (3.13) to both $u$ and $-u$, we obtain Theorem B of the introduction, with $\alpha, \beta, \alpha^{\prime}, \beta^{\prime}$ taken there to be $r, s, \varrho, \sigma$.

In order to prove Harnack's inequality we need a mean value inequality for $u^{p}$ when $-\infty<p<\infty$ and $u$ is a non-negative solution.

We begin by noting that if we use (C.3) instead of (C.1) we can prove the following analogue of (3.11):

Lemma 3.14. Suppose (3.1) holds, $0<m<u(x, t) \leq M<\infty$ in $R=R_{r, s}$, $r / 2<\varrho<r, s / 2<\sigma<s$ and $\epsilon>0,(1+\epsilon) \varrho<r$. Then, if $p>1$ and $u$ is a subsolution in $R$, or if $p<0$ and $u$ is a supersolution in $R$,

$$
\begin{aligned}
& \left(\iint_{R_{+}^{\prime}} u^{p h}\left(\frac{w_{2}}{w_{2}\left(Q_{r}\right)}+\frac{v}{v\left(Q_{r}\right)}\right) d x d t\right)^{1 / h} \\
& \leq c \epsilon^{-b} \frac{r^{2} s}{(r-(1+\epsilon) \varrho)^{2}(s-\sigma)}\left(1+\frac{p}{p-1} \frac{s}{r^{2}} \Lambda\left(Q_{r}\right)\right)\left(1+\frac{p}{p-1} \frac{r^{2}}{s} \frac{1}{\lambda\left(Q_{r}\right)}\right)^{1 / h} \\
& \cdot \iint_{R} u^{p}\left(\frac{p}{p-1} \frac{s}{r^{2}} w_{2}+v\right) d x d t .
\end{aligned}
$$

Moreover, if $0<p<1$ and $u$ is a supersolution in $R$, then

$$
\begin{aligned}
& \left(\iint_{R_{-}^{\prime}} u^{p h}\left(\frac{w_{1}}{w_{2}\left(Q_{r}\right)}+\frac{v}{v\left(Q_{r}\right)}\right) d x d t\right)^{1 / h} \\
& \leq c \epsilon^{-b} \frac{r^{2} s}{(r-(1+\epsilon) \varrho)^{2}(s-\sigma)}\left(1+\frac{p}{|p-1|}-\frac{s}{r^{2}} \Lambda\left(Q_{r}\right)\right)\left(1+\frac{p}{|p-1|} \frac{r^{2}}{s} \frac{1}{\lambda\left(Q_{r}\right)}\right)^{1 / h} \\
& \cdot \iint_{R} u^{p}\left(\frac{p}{|p-1|} \frac{s}{r^{2}} w_{2}+v\right) d x d t .
\end{aligned}
$$


Both inequalities are still true if we replace the integral averages on the right by the larger integral average

$$
\iint_{R} u^{p}\left(\frac{w_{2}}{w_{2}\left(Q_{r}\right)}+\frac{v}{v\left(Q_{r}\right)}\right) d x d t
$$

Theorem 3.15. Assume (3.1) holds, $r, s>0, r / 2<\varrho<r, s / 2<\sigma<s$. If $u$ is a non negative solution of (1.1) in $R$, then for $p>0$

ess $\sup _{R^{\prime}} u^{p}$

$$
\leq C^{c}\left(1+p \frac{s}{r^{2}} \Lambda\left(Q_{r}\right)\right)^{\frac{h}{h-1}}\left(1+p \frac{r^{2}}{s} \frac{1}{\lambda\left(Q_{r}\right)}\right)^{\frac{1}{h-1}} \iint_{R} u_{+}^{p}\left(\frac{w_{2}}{w_{2}\left(Q_{r}\right)}+\frac{v}{v\left(Q_{r}\right)}\right) d x d t,
$$

and for $p<0$

ess $\sup _{R_{+}^{\prime}} u^{p} \leq C^{\frac{h}{h-1}}\left(1+|p| \frac{s}{r^{2}} \Lambda\left(Q_{r}\right)\right)^{\frac{h}{h-1}}$

$$
\cdot\left(1+|p| \frac{r^{2}}{s}-\frac{1}{\lambda\left(Q_{r}\right)}\right)^{\frac{h}{h-1}} \iint_{R} u^{p}\left(\frac{w_{2}}{w_{2}\left(Q_{r}\right)}+\frac{v}{v\left(Q_{r}\right)}-\right) d x d t,
$$

where $C$ is given by (3.2).

Proof: In Lemma 3.17 of [GW2] we replace (3.20) by the result given here in Lemma 3.14 and then argue as in Lemma 3.17 of [GW2].

\section{Proof of Theorem $\mathbf{E}$}

We start with the following lemma.

Lemma 4.1. Suppose $Q=Q(\xi, r)$ and $\varphi$ is a $C^{1}$ function such that $\varphi \equiv 1$ in $k Q=Q(\xi, k r), 0<k<1,0 \leq \varphi \leq 1$, supp $\varphi \subset Q$ and

$$
\varphi(x) \varphi\left(H\left(t_{0}, x, y\right)\right) \leq \varphi(H(t, x, y))
$$

for all $x, y, t, t_{0}$ with $0 \leq t \leq t_{0}$. If $u$ is a Lipschitz function, 
$E=\{x \in Q(\xi, k r): u(x)=0\}$ and $|E| \geq \beta|Q|$ for some $0<\beta<1$, then if $x \in Q$,

$$
|u(x)| \sqrt{\varphi(x)} \leq c \int_{Q}\left|\nabla_{\lambda} u(z)\right| \sqrt{\varphi(z)} \frac{\delta(x, z)}{|Q(x, \delta(x, z))|} d z
$$

where $c$ is independent of $Q, u, x$.

PROOF: (The general outline of this proof follows the steps of the proof of Lemma 4.3 in [FS].) If $x \in Q=Q(\xi, r)$ then $Q(\xi, r) \subset Q\left(x, 2 a^{2} r\right)$ and $Q(x$, $r) \subset Q\left(\xi, 2 a^{2} r\right)$. Therefore, by doubling, $|Q(x, r)| \simeq|Q|$. Now, we note that there exists $\sigma \in\{-1,1\}^{n}$ such that $\left|E \cap Q^{\sigma}\left(x, 2 a^{2} r\right)\right| \geq c \beta\left|Q^{\sigma}\left(x, 2 a^{2} r\right)\right|$. In fact, $E=\cup_{\sigma}\left(Q^{\sigma}\left(x, 2 a^{2} r\right) \cap E\right)$ and so there exists $\sigma$ such that

$$
\left|Q^{\sigma}\left(x, 2 a^{2} r\right) \bigcap E\right| \geq \beta 2^{-n}|Q| \geq c \beta\left|Q^{\sigma}\left(x, 2 a^{2} r\right)\right| .
$$

We also claim that there exist $\alpha, \epsilon \in \mathbf{R}^{n}$, independent of $x$ and $r, 0<\epsilon_{j}<$ $\alpha_{j}, j=1, \ldots, n$, such that

$$
\left|E \cap Q^{\sigma}\left(x, 2 a^{2} r\right) \bigcap H\left(2 a^{2} r, x, \Delta_{\epsilon}^{\alpha}(\sigma)\right)\right| \geq \frac{c \beta}{2}\left|Q^{\sigma}\left(x, 2 a^{2} r\right)\right| .
$$

To prove this fact, apply (1.14) to $\gamma=\frac{c \beta}{2}$ and find $\alpha, \epsilon \in \mathbf{R}^{n}, 0<\epsilon_{j}<\alpha_{j}$, $j=1, \ldots, n$, such that

$$
\left|H\left(2 a^{2} r, x, \Delta_{\epsilon}^{\alpha}(\sigma)\right) \cap Q^{\sigma}\left(x, 2 a^{2} r\right)\right| \geq\left(1-\frac{c \beta}{2}\right)\left|Q^{\sigma}\left(x, 2 a^{2} r\right)\right| .
$$

Then,

$$
\begin{aligned}
& \left|Q^{\sigma}\left(x, 2 a^{2} r\right)\right| \geq\left|\left(Q^{\sigma}\left(x, 2 a^{2} r\right) \cap E\right) \cup\left(Q^{\sigma}\left(x, 2 a^{2} r\right) \cap H(\ldots)\right)\right|= \\
& \left|Q^{\sigma}\left(x, 2 a^{2} r\right) \cap E\right|+\left|Q^{\sigma}\left(x, 2 a^{2} r\right) \cap H(\ldots)\right|-\left|E \cap Q^{\sigma}\left(x, 2 a^{2} r\right) \cap H(\ldots)\right| \\
& \geq\left|Q^{\sigma}\left(x, 2 a^{2} r\right)\right|\left(c \beta+1-\frac{C \beta}{2}\right)-\left|E \cap Q^{\sigma}\left(x, 2 a^{2} r\right) \bigcap H(\ldots)\right|
\end{aligned}
$$

and therefore the claim follows.

We can assume $x \notin E$ and define $\Sigma=\left\{y \in \Delta_{\epsilon}^{\alpha}(\sigma): H\left(2 a^{2} r, x, y\right) \in E\right\}$. Let $K$ be a smooth function supported in $\Delta_{\epsilon / 2}^{2 \alpha}(\sigma), 0 \leq K \leq 1, K \equiv 1$ on $\Delta^{\alpha}{ }_{\epsilon}(\sigma)$. Suppose $u \in \operatorname{Lip}(Q)$. If $y \in \Sigma$ then

$$
|u(x)| \sqrt{\varphi(x)}=\left|u(x)-u\left(H\left(2 a^{2} r, x, y\right)\right)\right| K(y) \sqrt{\varphi(x)},
$$

and if we integrate on $\Sigma$, we obtain 


$$
|u(x)| \sqrt{\varphi(x)}|\Sigma|=\int_{\Sigma}\left|u(x)-u\left(H\left(2 a^{2} r, x, y\right)\right)\right| K(y) \sqrt{\varphi(x)} d y .
$$

Now we note that $\varphi\left(H\left(2 a^{2} r, x, y\right)\right)=1$ if $y \in \Sigma$ and using (4.2) we get $\varphi(x) \leq \varphi(H(t, x, y))$ for any $0 \leq t \leq 2 a^{2} r$. Therefore,

$$
\begin{aligned}
& |u(x)| \sqrt{\varphi(x)}|\Sigma| \leq \int_{\text {supp } K}\left|\int_{0}^{2 a^{2} r} \frac{d}{d t}(u(H(t, x, y))) d t\right| \sqrt{\varphi(H(t, x, y))} d y \\
& \leq \int_{\text {supp } K}\left|\int_{0}^{2 a^{2} r}\langle\nabla u(H(t, x, y)), \dot{H}(t, x, y)\rangle d t\right| \sqrt{\varphi(H(t, x, y))} d y \\
& \leq \int_{0}^{2 a^{2} r} \int_{\text {supp } K}\left|\nabla_{\lambda} u(H(t, x, y))\right||y| \sqrt{\varphi(H(t, x, y))} d y d t .
\end{aligned}
$$

If we make change of variables $z=H(t, x, y)$ in $\Delta_{\epsilon / 2}^{2 \alpha}(\sigma)$, then

$$
\left|\operatorname{det} \frac{\partial z}{\partial y}(t, x, y)\right|=\Pi_{j=1}^{n} \int_{0}^{t} \lambda_{j}(H(s, x, y)) d s .
$$

For $y \in \Delta_{\epsilon / 2}^{2 \alpha}(\sigma)$, the last product is equivalent to $|Q(x, t)|$ by (1.15). Hence (4.6) $|u(x)| \sqrt{\varphi(x)} \leq \frac{c}{|\Sigma|} \int_{0}^{2 a^{2} r} \frac{1}{|Q(x, t)|} \int_{\mathrm{H}\left(t, x, \Delta_{\epsilon / 2}^{2 \alpha}(\sigma)\right)}\left|\nabla_{\lambda} u(z)\right| \sqrt{\varphi(z)} d z d t$.

Note that there exists $c>0$ such that $H\left(t, x, \Delta_{\epsilon / 2}^{2 \alpha}(\sigma)\right) \subset Q(x, c t)$. In fact, if we define $\gamma(s)=H(s /|y|, x, y)$ then

$$
\begin{aligned}
\langle\dot{y}(s), \xi\rangle^{2} & =\left\{\sum_{j=1}^{n} \lambda_{j}\left(H\left(\frac{s}{|y|}, x, y\right)\right) y_{j} \xi_{j}\right\}^{2} \frac{1}{|y|^{2}} \\
& \leq \sum_{j=1}^{n} \lambda_{j}^{2}\left(H\left(\frac{s}{|y|}, x, y\right)\right) \xi_{j}^{2} \\
& =\sum_{j=1}^{n} \lambda_{j}(\gamma(s)) \xi_{j}^{2}
\end{aligned}
$$

for every $\xi \in R^{n}$. So, $\gamma$ is a $\lambda$-subunit curve starting from $x$ and attaining $H(t, x, y)$ at the time $s=t|y|$. Therefore by (1.9),

$$
\delta(x, H(t, x, y)) \leq a d(x, H(t, x, y)) \leq a t|y| \leq c t
$$

where $c=2 \alpha a$ 
Thus, from (4.6), we obtain

$$
|u(x)| \sqrt{\varphi(x)} \leq \frac{c}{|\Sigma|} \int_{0}^{2 a^{2} r} \frac{1}{|Q(x, t)|} \int_{Q(x, c t)}\left|\nabla_{\lambda} u(z)\right| \sqrt{\varphi(z)} d z d t
$$

and, interchanging the order of integration and using the fact that supp $\varphi \subset Q$ (the argument we are going to present next is due to Chanillo, Sawyer and Wheeden), we get

$$
|u(x)| \sqrt{\varphi(x)} \leq \frac{c}{|\Sigma|} \int_{Q}\left|\nabla_{\lambda} u(z)\right| \sqrt{\varphi(z)}\left(\int_{c \delta(x, z)}^{\infty} \frac{d t}{|Q(x, t)|}\right) d z
$$

We claim that $\int_{c h}^{\infty} \frac{d t}{|Q(x, t)|} \leq c \frac{c h}{|Q(x, h)|}$. To prove this we note that, by (1.8),

$$
\frac{|Q(x, t)|}{t}=\prod_{j=2}^{n} F_{j}\left(x^{*}, t\right)
$$

and consequently by (1.10), there exists $\epsilon>0$ such that if $t>\tau$ then

$$
\frac{|Q(x, t)|}{t} \geq c\left(\frac{t}{\tau}\right)^{\epsilon} \frac{|Q(x, \tau)|}{\tau} .
$$

Hence,

$$
\int_{c h}^{\infty} \frac{d t}{|Q(x, t)|}=\int_{c h}^{\infty} \frac{t}{|Q(x, t)|} \frac{d t}{t} \leq \int_{c h}^{\infty} \frac{h}{|Q(x, h)|}\left(\frac{h}{t}\right)^{\epsilon} \frac{d t}{t}=c \frac{h}{|Q(x, h)|} .
$$

Finally, we note that $|\Sigma| \geq c>0$, with $c$ independent of $x$, since, by the change of variables $z=H\left(2 a^{2} r, x, y\right)$,

$$
\begin{aligned}
|\Sigma| & =\int_{\Sigma} d y \simeq \int_{H\left(2 a^{2} r, x, \Sigma\right)} \frac{1}{\mid Q\left(x, \frac{\left.2 a^{2} r\right) \mid}{} d z\right.} \\
& =\frac{\left|H\left(2 a^{2} r, x, \Sigma\right)\right|}{\left|Q\left(x, 2 a^{2} r\right)\right|}=\frac{\left|E \bigcap H\left(2 a^{2} r, x, \Delta_{\epsilon}^{\alpha}(\sigma)\right)\right|}{\left|Q\left(x, 2 a^{2} r\right)\right|} \\
& \geq c \beta \frac{\left|Q^{\sigma}\left(x, 2 a^{2} r\right)\right|}{Q\left(x, 2 a^{2} r\right) \mid} \geq c>0 .
\end{aligned}
$$

The lemma follows by combining the last two last estimates with (4.7). 
ProOF OF THEOREM E.

Define $T f(x)=\int_{\mathbf{R}^{n}} f(y) K(x, y) d y$, where $K(x, y)=\frac{\delta(x, y)}{|Q(x, \delta(x, y))|}$.

Fix $S$ a $d$-ball. In order to show that for a pair of weights $\tilde{\nu}, \tilde{w}$ we have $\|T f\|_{L^{2}(S, \tilde{v})} \leq\|f\|_{L^{2}(S, \tilde{w})}$ (where $\|f\|_{L^{2}(S, \tilde{v})}=\left(\int_{S} f^{2} \tilde{v}\right)^{1 / 2}$ ) for all $f \geq 0$, supp $f \subset S$, according to [SW], we need to verify that the following ronditions hold:

(a) there exists $s>1$ such that

$$
\varphi(I)|I|\left(\frac{1}{|I|} \int_{I} \tilde{v}^{s} d x\right)^{\frac{1}{2 s}}\left(\frac{1}{|I|} \int_{I} \tilde{w}^{-s} d x\right)^{\frac{1}{2 s}} \leq c
$$

for all $d$-balls $I \subset 2 S$, where $\varphi(I)$ is defined to be

$$
\varphi(I)=\sup \left\{K(x, y): x, y \in I, d(x, y) \geq \frac{1}{2} r(I)\right\}
$$

(b) there is $\epsilon>0$ such that

$$
\frac{|I|}{|I|} \leq c_{\epsilon} \frac{\varphi(I)}{\varphi\left(I^{\prime}\right)}\left(\frac{r\left(I^{\prime}\right)}{r(I)}\right)^{\epsilon}
$$

for all pairs of $d$-balls $I^{\prime} \subset I$.

Note that it is convenient to work with $d$ since the results of $[\mathrm{SW}]$ hold for pseudo-metrics (a pseudo-metric $d$ is a quasi-metric satisfying $d(x, y)=d(y, x)$ for all $x, y \in \mathbf{R}^{n}$ ).

Define $\tilde{v}=\frac{v}{v(S)}$ and $\tilde{w}=\frac{w_{1}}{w_{1}(S)} r(S)^{2}$. Note that if $x, y \in I$ and $d(x, y) \geq \frac{1}{2} r(I)$, then by (1.9)

$$
K(x, y)=\frac{\delta(x, y)}{|Q(x, \delta(x, y))|} \leq \frac{2 \operatorname{ar}(I)}{\left|Q\left(x, \frac{1}{2 a} r(I)\right)\right|} \leq c \frac{r(I)}{|Q(x, r(I))|}
$$


and since $x \in I,|Q(x, r(I))| \simeq|I|$. Therefore,

$$
\varphi(I) \leq c \frac{r(I)}{|I|} .
$$

So, the expression in (a) is bounded by

$$
\begin{aligned}
& c \frac{r(I)}{|I|}|I|\left(\frac{1}{|I|} \int_{I}\left(\frac{v}{v(S)}\right)^{s} d x\right)^{\frac{1}{2 s}}\left(\frac{1}{|I|} \int_{I}\left(\frac{w_{1}}{w_{1}(S)} r(S)^{2}\right)^{-s} d x\right)^{\frac{1}{2 s}} \\
& \leq c \frac{r(I)}{r(S)}\left(\frac{1}{|I|} \int_{I}\left(\frac{v}{v(S)}\right)^{s} d x\right)^{\frac{1}{2 s}}\left(\frac{1}{|I|} \int_{I}\left(\frac{w_{1}}{w_{1}(S)}\right)^{-s} d x\right)^{\frac{1}{2 s}},
\end{aligned}
$$

which is equivalent to the expression in condition (1.18) (if we use doubling and (1.9)). This proves (a).

To show (b) we note that if $x, y \in I$ and $d(x, y) \geq \frac{1}{2} r(I)$ then

$$
K(x, y) \geq \frac{(2 a)^{-1} r(I)}{|Q(x, 2 \operatorname{ar}(I))|} \geq c \frac{r(I)}{|I|} .
$$

Thus $\varphi(I) \simeq \frac{r(I)}{|I|}$. Then, if $I^{\prime} \subset I, \frac{\varphi(I)}{\varphi\left(I^{\prime}\right)} \simeq \frac{r(I)}{r\left(I^{\prime}\right)} \frac{\left|I^{\prime}\right|}{|I|}$ and we obtain (b) with $\epsilon=1$.

By doubling and (1.9), it follows that

$$
\|T f\|_{L^{2}(Q, \tilde{\nu})} \leq c\|f\|_{L^{2}(Q, \tilde{w})}
$$

for all $f \geq 0, \operatorname{supp} f \subset Q$, where $\tilde{v}=\frac{v}{v(Q)}$ and $\tilde{w}=\frac{w_{1}}{w_{1}(Q)} r(Q)^{2}$.

Suppose $u$ is a Lipschitz function in $Q$ and $|E|=\mid\{x \in Q(\xi, k r): u(x)$ $=0\}|\geq \beta| Q \mid, 1 / 2<k<1$. If we combine Lemma 4.1 and the fact that $\|T f\|_{L^{2}(Q, \tilde{v})} \leq c\|f\|_{L^{2}(Q, \tilde{w})}$ we obtain

$$
\begin{aligned}
& \left(\frac{1}{v(Q)} \int_{Q}|u(x)|^{2} \varphi(x) v(x) d x\right)^{1 / 2} \\
& \leq \operatorname{cr}(Q)\left(\frac{1}{w_{1}(Q)} \int_{Q}\left|\nabla_{\lambda} u(z)\right|^{2} \varphi(z) w_{1}(z) d z\right)^{1 / 2}
\end{aligned}
$$


Given $Q$ and a general Lipschitz function $u$, there is a number $\mu=\mu(u, Q)$, the media of $u$ in $Q$, such that if $Q^{+}=\{x \in Q: u(x) \geq \mu\}$ and $Q^{-}=\{x \in Q$ : $u(x) \leq \mu\}$ then $\left|Q^{+}\right| \geq|Q| / 2$ and $\left|Q^{-}\right| \geq|Q| / 2$. Hence, $u_{1}=$ $\max \{u-\mu(u, k Q), 0\}$ and $u_{2}=\max \{\mu(u, k Q)-u, 0\}$ satisfy the hypohesis of Lemma (4.1) for some $\beta$ depending on $k$ and so if we apply (4.8) to $u_{1}$ and $u_{2}$ and add both inequalities, we get

(4.9) $\int_{Q}|u(x)-\mu|^{2} \varphi(x) v(x) d x \leq \operatorname{cr}(Q)^{2} \frac{v(Q)}{w_{1}(Q)} \int_{Q} \mid \nabla_{\lambda} u(z)^{2} \varphi(z) w_{1}(z) d z$.

Finally, it is easy to see that in (4.9) $\mu$ can be replaced by the average $A_{Q}$ of $u$ defined in Theorem E. In fact,

$$
\begin{aligned}
\int_{Q}\left|u(x)-A_{Q}\right|^{2} \varphi(x) v(x) d x \\
\quad \leq 2 \int_{Q}|u(x)-\mu|^{2} \varphi(x) v(x) d x \\
\quad+2 \int_{Q}\left|\mu-A_{Q}\right|^{2} \varphi(x) v(x) d x
\end{aligned}
$$

and

$$
\begin{aligned}
\int_{Q}\left|\mu-A_{Q}\right|^{2} \varphi(x) v(x) d x & =(\varphi v)(Q)\left|\mu-A_{Q}\right|^{2} \\
& =(\varphi v)(Q)\left|\mu-\frac{1}{\varphi(Q)} \int_{Q} u(x) \varphi(x) d x\right|^{2} \\
& \leq(\varphi v)(Q)\left(\frac{1}{\varphi(Q)} \int_{Q}|u(x)-\mu| \varphi(x) d x\right)^{2} \\
& \leq \frac{(\varphi v)(Q)}{(\varphi(Q))^{2}} \int_{Q}|u(x)-\mu|^{2} \varphi^{2}(x) v(x) d x \int_{Q} \frac{1}{v(x)} d x,
\end{aligned}
$$

where in the last inequality we used Schwarz's inequality. Since $v \in A_{2}$ and $0 \leq \varphi \leq 1$, it follows from (4.9) and (4.10) that

$$
\begin{aligned}
\int_{Q}\left|u(x)-A_{Q}\right|^{2} \varphi(x) v(x) d x & \\
\leq & \operatorname{cr}(Q)^{2}\left[1+\left(\frac{|Q|}{\varphi(Q)}\right)^{2}\right] \frac{v(Q)}{w_{1}(Q)} \int_{Q}\left|\nabla_{\lambda} u(z)\right|^{2} \varphi(z) w_{1}(z) d z .
\end{aligned}
$$


This finishes the proof of Theorem $\mathrm{E}$ if we note that $\varphi(Q) \simeq|Q|$ since $1 / 2 \leq k \leq 1$.

The next corollary is also helpful.

Corollary 4.11. Theorem $E$ is also true with $A_{Q}=\frac{1}{(\varphi v)(Q)} \int_{Q} u \varphi v d x$. Just note that

$$
\begin{aligned}
\int_{Q}\left|\mu-A_{Q}\right|^{2} \varphi v d x & =(\varphi v)(Q)\left|\mu-A_{Q}\right|^{2} \\
& \leq(\varphi v)(Q)\left(\frac{1}{(\varphi v)(Q)} \int_{Q}|\mu-u| \varphi v d x\right)^{2} \\
& \leq \int_{Q}|\mu-u|^{2} \varphi v d x
\end{aligned}
$$

where the last inequality follows by Schwarz's inequality.

\section{Harnack's inequality}

The proof of Theorem A follows as an application of Bombieri's lemma which we state next. For its proof see Section 5 of [GW2].

Lemma 5.1. Let $R(\varrho)$ be a one parameter family of rectangles in $\mathbf{R}^{n+1}$, $R(\sigma) \subset R(\varrho), 1 / 2 \leq \sigma \leq \varrho \leq 1$ and let $\nu$ be a doubling measure in $\mathbf{R}^{n+1}$. Let $A, \mu, M, m, \theta$ and $\varkappa$ be positive constants such that $M \geq 1 / \mu$ and suppose that $f$ is a positive measurable function defined in a neighborhood of $R(1)$ satisfying

$$
\text { ess } \sup _{R(\sigma)} f^{p} \leq \frac{A}{(\varrho-\sigma)^{m}} \iint_{R(\varrho)} f^{p} \nu(x) d x d t
$$

for all $\sigma, \varrho, p, 1 / 2 \leq \theta \leq \sigma<\varrho<1,0<p<M$ and

$$
\nu(\{(x, t) \in R(1): \log f>s\}) \leq\left(\frac{\mu}{s}\right)^{x} \nu(R(1))
$$


for all $s>0$. Then there is a constant $\gamma=\gamma(A, m, \varkappa)>0$ such that

$$
\log \left(\operatorname{ess} \sup _{R(\theta)} u\right) \leq \frac{\gamma}{(1-\theta)^{2 m}} \mu .
$$

Hence, in order to prove Theorem A, we need a mean value inequality (that we proved in Section 3) and a logarithm estimate which is given by Theorem F (some steps of its proof we will present in this section). The next lemma shows that the test function described on page 537 of [FL1] satisfies the conditions of Theorem E. Then, as we said before, the proof of Theorem $\mathrm{F}$ follows as Lemma 4.9 of [GW2].

Lemma 5.4. Given $Q=Q(\xi, r)$ and $0<k<1$, there exists $\varphi \in C^{1}$ such that $\varphi \equiv 1$ in $k Q, 0 \leq \varphi \leq 1$, $\operatorname{supp} \varphi \subset Q,\left|\nabla_{\lambda} \varphi\right| \leq \frac{c}{r(1-k)}$ and $\varphi(x) \cdot \varphi\left(H\left(t_{0}, x, y\right)\right) \leq \varphi(H(t, x, y))$ for all $x, y, t, t_{0}$ with $0 \leq t \leq t_{0}$.

Proof: Consider the function $\varphi$ given by [FL1], page 537:

$$
\varphi(x)=\prod_{j=1}^{n} \psi\left(\frac{\left|x_{j}-\xi_{j}\right|}{F_{j}\left(\xi^{*}, r\right)}\right)
$$

where $\psi \in C^{\infty}(\mathbf{R}), 0 \leq \psi \leq 1, \psi(t)=\psi(-t), \psi \equiv 1$ on $[-k, k], \psi=0$ outside ]$-1,1\left[,\left|\psi^{\prime}(t)\right| \leq 2(1-k)^{-1}\right.$, for all $t \in \mathbf{R}$. Here, we show that $\varphi$ satisfies the last condition since all the others are proved in [FL1], page 537.

Fix $t, 0<t<t_{0}, x$ and $y$. Define $z=H(t, x, y)$. Then,

$$
z_{j}=x_{j}+y_{j} \int_{0}^{t} \lambda_{j}(H(s, x, y)) d s .
$$

Suppose $z_{j}-\xi_{j} \geq 0$. If $y_{j} \geq 0$ then

$$
\left|z_{j}-\xi_{j}\right| \leq x_{j}-\xi_{j}+y_{j} \int_{0}^{t_{0}} \lambda_{j}(H(s, x, y)) d s=H_{j}\left(t_{0}, x, y\right)-\xi_{j} .
$$

On the other hand, if $y_{j}<0$,

$$
\left|z_{j}-\xi_{j}\right| \leq\left|x_{j}-\xi_{j}\right| \text {. }
$$

Thus, if $z_{j}-\xi_{j} \geq 0$ then $\left|z_{j}-\xi_{j}\right| \leq\left|H_{j}\left(t_{0}, x, y\right)-\xi_{j}\right|$ or $\left|z_{j}-\xi_{j}\right|$ $\leq\left|x_{j}-\xi_{j}\right|$. The same holds if $z_{j}-\xi_{j}<0$. Since $\psi(t)$ can be chosen to be non-increasing for positive $t$, then $\varphi(z) \geq a_{1} \ldots a_{n}$, where

$$
a_{j}=\psi\left(\frac{\left|x_{j}-\xi_{j}\right|}{F_{j}\left(\xi^{*}, r\right)}\right)
$$


or

$$
a_{j}=\psi\left(\frac{\left|H_{j}\left(t_{0}, x, y\right)-\xi_{j}\right|}{F_{j}\left(\xi^{*}, r\right)}\right)
$$

Since $0 \leq \psi \leq 1$,

$$
a_{j} \geq \psi\left(\frac{\left|H_{j}\left(t_{0}, x, y\right)-\xi_{j}\right|}{F_{j}\left(\xi^{*}, r\right)}\right) \psi\left(\frac{\left|x_{j}-\xi_{j}\right|}{F_{j}\left(\xi^{*}, r\right)}\right)
$$

for $1 \leq j \leq n$. Therefore,

$$
\varphi(z) \geq \varphi(x) \varphi\left(H\left(t_{0}, x, y\right)\right) .
$$

The next three lemmas are needed in order to show that the hypothesis in Theorem A imply those in Theorems D and E.

Lemma 5.5. Assume that Poincaré's inequality holds for $w_{1}, w_{2}$ with $q=2$ and $\mu=1$. Then

$$
\left(\frac{r(I)}{r(B)}\right)^{2} \frac{w_{2}(I)}{w_{2}(B)} \leq c \frac{w_{1}(I)}{w_{1}(B)}
$$

for any pair of $\delta$-balls $I, B$, with $I \subset 2 B$.

ProOF: Suppose $I=Q\left(u_{0}, r(I)\right)$ and $B=Q(x, r(B))$ and define

$$
F(u)=\sum_{j=1}^{n} \frac{\left|u_{j}-\left(u_{0}\right)_{j}\right|}{F_{j}\left(u_{0}^{*}, r(I)\right)} r(I) \varphi(u)
$$

where $\varphi$ is the function described in lemma (5.4) associated with $I$ (as opposed to $B$ ) and $k=1 / 2$. If $u \in I$, by (1.8)

$$
\left|\frac{\partial F}{\partial u_{k}}(u)\right| \leq \frac{r(I)}{F_{k}\left(u_{0}^{*}, r(I)\right)}+\frac{\partial \varphi}{\partial u_{k}}(u) n r(I)
$$

for $k \in\{1, \ldots n\}$, and using the fact that $\lambda_{k}(u)=\lambda_{k}\left(u^{*}\right) \leq \lambda_{k}\left(H\left(u^{*}, r(I)\right)\right)$ if $u \in I$ we get

$$
\left|\lambda_{k}(u) \frac{\partial F}{\partial u_{k}}(u)\right| \leq \frac{F_{k}\left(u^{*}, r(I)\right)}{F_{k}\left(u_{0}^{*}, r(I)\right)}+n r(I) \lambda_{k}(u) \frac{\partial \varphi}{\partial u_{k}}(u)
$$


and by Lemma 2.4 and the fact that $\left|\nabla_{\lambda} \varphi\right| \leq c / r(I)$ we have $\left|\nabla_{\lambda} F(u)\right| \leq c \chi_{I}$.

We have Poincaré's inequality for $F$, i.e.,

$$
\begin{aligned}
& \left(\frac{1}{w_{2}(B)} \int_{n 4^{\eta+1} B}\left|F(u)-a v_{n 4^{\eta+1} B} F\right|^{2} w_{2}(u) d u\right)^{1 / 2} \\
& \quad \leq \operatorname{cr}(B)\left(\frac{1}{w_{1}(B)} \int_{n a^{2} 4^{\eta+1} B}\left|\nabla_{\lambda} F(u)\right|^{2} w_{1}(u) d u\right)^{1 / 2},
\end{aligned}
$$

where $\eta=\max _{j=1, \ldots n}\left\{G_{j}\right\}$. The right side of (5.6) is bounded by $\operatorname{cr}(B)\left(\frac{w_{1}(I)}{w_{1}(B)}\right)^{1 / 2}$ by doubling and the fact that $\left|\nabla_{\lambda} F\right| \leq c \chi_{I}$. Now, if $u \notin \frac{1}{4} I$ there exists $k \in\{1, \ldots, n\}$ such that

$$
\left|u_{k}-\left(u_{0}\right)_{k}\right| \geq F_{k}\left(u_{0}^{*}, \frac{1}{4} r(I)\right)
$$

and then if $u \in \frac{1}{2} I \backslash \frac{1}{4} I$ (note that $\varphi(u)=1$ )

$$
F(u) \geq \frac{F_{k}\left(u^{*}, \frac{1}{4} r(I)\right)}{F_{k}\left(u_{0}^{*}, r(I)\right)} r(I) \geq\left(\frac{1}{4}\right)^{G_{k}} r(I) \geq \frac{1}{4^{\eta}} r(I) .
$$

Also, if $u \in I, F(u) \leq n r(I)$ and therefore

$$
a v_{n 4^{\eta+1} B} F \leq \frac{|I|}{\left|n 4^{\eta+1} B\right|} n r(I) .
$$

But, by (1.10), $F_{j}\left(x_{B}^{*}, n 4^{\eta+1} r(B)\right) \geq 2 n 4^{\eta} F_{j}\left(x_{B}^{*}, 2 r(B)\right)$, and by (1.11),

$$
\left|n 4^{\eta+1} B\right| \geq\left(2 n 4^{\eta}\right)^{n}|2 B| \geq 2 n 4^{\eta}|2 B| \text {. }
$$

Hence, since $I \subset 2 B, a v_{n 4^{7+1} B} F \leq r(I) / 2 \cdot 4^{\eta}$ and then if $u \in \frac{1}{2} I \backslash \frac{1}{4} I$ (using also 5.7)),

$$
\left|F(u)-a v_{n^{4 \eta+1} B} F\right| \geq c r(I) .
$$

Therefore, the left hand side of (5.6) is larger than a constant times 


$$
\left[\frac{(r(I))^{2}}{w_{2}(B)} w_{2}\left(\frac{1}{2} I \backslash \frac{1}{4} I\right)\right]^{1 / 2} \geq \operatorname{cr}(I)\left(\frac{w_{2}(I)}{w_{2}(B)}\right)^{1 / 2}
$$

where in the last inequality we used the fact that $w_{2}\left(\frac{1}{2} I \backslash \frac{1}{4} I\right) \simeq w_{2}(I)$, which is shown in the next lemma.

Lemma 5.8. If $w$ is a doubling weight then $W(Q(u, 2 s) \backslash Q(u, s))$ is equivalent to $w(Q(u, s))$.

Proof: Choose $\eta \in Q(u, 2 s)$ such that $\delta(u, \eta)=\frac{3 s}{2}$. By Lemma 2.5,

$$
Q\left(\eta, \frac{3 \epsilon s}{2\left(2 a^{2}\right)^{5}}\right) \subset Q\left(u,(1+\epsilon) \frac{3 s}{2}\right)
$$

for any $0<\epsilon<1$.

Choose $j$ such that $\delta(u, \eta)=\varphi_{j}\left(u^{*},\left|\eta_{j}-u_{j}\right|\right)$. Then, if $y \in Q\left(\eta, \frac{3 \epsilon s}{2\left(2 a^{2}\right)^{\zeta}}\right)$,

$$
\begin{aligned}
F_{j}\left(u^{*}, \frac{3 s}{2}\right)= & \left|\eta_{j}-u_{j}\right| \leq\left|\eta_{j}-y_{j}\right|+\left|y_{j}-u_{j}\right| \\
& \leq F_{j}\left(\eta^{*}, \frac{3 \epsilon s}{2\left(2 a^{2}\right)^{\zeta}}\right)+\left|y_{j}-u_{j}\right|
\end{aligned}
$$

By (1.10) and Lemma 2.4,

$$
F_{j}\left(u^{*}, \frac{3 s}{2}\right) \leq \epsilon F_{j}\left(u^{*}, \frac{3 s}{2}\right)+\left|y_{j}-u_{j}\right|
$$

Thus,

$$
\left|y_{j}-u_{j}\right| \geq(1-\epsilon) F_{j}\left(u^{*}, \frac{3 s}{2}\right) \geq F_{j}\left(u^{*},(1-\epsilon) \frac{3 s}{2}\right) .
$$

If we choose $\epsilon=1 / 3$ we have proved that

$$
Q\left(\eta, \frac{s}{2\left(2 a^{2}\right)^{\zeta}}\right) \subset Q(u, 2 s) \backslash Q(u, s) .
$$

The lemma follows by doubling. 
Lemma 5.9. If $w_{1} \in A_{2}, v \in A_{\infty}$ and Poincaré's inequality holds for $w_{1}$, $v$ with $q=2$ and $\mu=1$, then condition (1.21) holds.

Proof: If $v \in A_{\infty}$ there exists $s>1$ such that

$$
\left(\frac{1}{|I|} \int_{I}\left(\frac{v}{v(B)}\right)^{s} d x\right)^{1 / s} \leq \frac{1}{|I|} \frac{v(I)}{v(B)} .
$$

So, since Poincaré's inequality holds for $w_{1}, v$ with $q=2$, by Lemma 5.5

$$
\left(\frac{r(I)}{r(B)}\right)^{2}\left(\frac{1}{|I|} \int_{I}\left(\frac{v}{v(B)}\right)^{s} d x\right)^{1 / s} \leq c \frac{1}{|I|} \frac{w_{1}(I)}{w_{1}(B)}
$$

and the above condition is equivalent to condition (1.18) since $w_{1} \in A_{2}$.

Now we are ready to prove Theorem A.

\section{Proof of THEOREM A}

Let $u$ be a non-negative solution of (1.1) in the cylinder $R_{\alpha, \beta}=R_{\alpha, \beta}\left(x_{0}, t_{0}\right)$ $=Q\left(x_{0}, \alpha\right) \times\left(t_{0}-\beta, t_{0}+\beta\right)$. If we define $T(x, t)=\left(x, \beta t+t_{0}\right)$ and $\bar{u}(x, t)=u(T(x, t))$ then $u$ is a solution in $R_{\alpha, 1}\left(x_{0}, 0\right)$ of the equation

$$
v(x) \ddot{u}_{t}=\operatorname{div}(\bar{A}(x, t) \nabla \bar{u}),
$$

where the coefficients matrix $\bar{A}=\left(a_{i j}\right)$ are defined by $\bar{a}_{i j}(x, t)=\beta a_{i j}\left(x, \beta t+t_{0}\right)$ and satisfies the degeneracy condition

$$
\bar{w}_{1}(x) \sum_{j=1}^{n} \lambda_{j}^{2}(x) \xi_{j}^{2} \leq \sum_{j=1}^{n} \bar{a}_{i j}(x, t) \xi_{i} \xi_{j} \leq \bar{w}_{2}(x) \sum_{j=1}^{n} \lambda_{j}^{2}(x) \xi_{j}^{2},
$$

if we put $\bar{w}_{i}=\beta w_{i}$, for $i=1,2$.

Suppose $|p|<\left[\alpha^{-2} \bar{\Lambda}\left(Q\left(x_{0}, \alpha\right)\right)+\alpha^{2} / \bar{\lambda}\left(Q\left(x_{0}, \alpha\right)\right)\right]^{-1}$, where $\bar{\Lambda}(Q)=$ $\bar{w}_{2}(Q) / v(Q), \bar{\lambda}(Q)=\bar{w}_{1}(Q) / v(Q)$. Write

$$
\begin{gathered}
R^{-}(\varrho)=Q\left(x_{0}, \frac{(\varrho+1) \alpha}{3}\right) \times\left(-\frac{1}{2}-\frac{\varrho}{2},-\frac{1}{2}+\frac{\varrho}{2}\right) \\
R^{+}(\varrho)=Q\left(x_{0}, \frac{(\varrho+1) \alpha}{3}\right) \times\left(\frac{1}{2}-\frac{\varrho}{2}, 1\right)
\end{gathered}
$$


If we take $1 / 2<\varrho<r \leq 1$ then the mean value inequalities in Theorem 3.15 applied to $u$ give

(5.10) $\operatorname{ess} \sup _{R^{-}(\varrho)} \bar{u}^{p} \leq c \frac{1}{(r-\varrho)^{m}} \iint_{R^{-}(r)} \bar{u}^{p}\left(\frac{\bar{w}_{2}}{\bar{w}_{2}\left(Q_{\alpha}\right)}+\frac{v}{v\left(Q_{\alpha}\right)}\right) d x d t$,

for some $m>0$, if $p>0$, where $Q_{\alpha}=Q\left(x_{0}, \alpha\right)$, and

$$
\text { ess } \sup _{R^{+}(\varrho)} \bar{u}^{p} \leq c \frac{1}{(r-\varrho)^{m}} \iint_{R^{+}(r)} \bar{u}^{p}\left(\frac{\bar{w}_{2}}{\bar{w}_{2}\left(Q_{\alpha}\right)}+\frac{v}{v\left(Q_{\alpha}\right)}\right) d x d t
$$

if $p<0$. Moreover, by Theorem $\mathrm{B}, \bar{u}$ is locally bounded and by adding $\epsilon>0$, we may assume by letting $\epsilon \rightarrow 0$ at the end of the proof that $\bar{u}$ is bounded below in $R_{\alpha, 1}\left(x_{0}, 0\right)$ by a positive constant.

Now, by Theorem $F$, we have

$$
\begin{aligned}
{\left[\left(\frac{v}{v\left(Q_{\alpha}\right)}\right.\right.} & \left.\left.+\frac{\bar{w}}{\bar{w}_{2}\left(Q_{\alpha}\right)}\right) \otimes 1\right]\left(E^{+}\right) \\
& \leq\left\{\frac{1}{s} \frac{v\left(Q_{\alpha}\right)}{\bar{w}_{1}\left(Q_{\alpha}\right)} \alpha^{2}\right\}^{\kappa} \\
& \leq c\left\{\frac{1}{s}\left[\alpha^{-2} \bar{\Lambda}\left(Q_{\alpha}\right)+\alpha^{2} \frac{1}{\bar{\lambda}\left(Q_{\alpha}\right)}\right]\right\}^{\varkappa},
\end{aligned}
$$

and the same inequality holds for $E^{-}$, where $E^{+}, E^{-}$are defined in Theorem $\mathrm{F}$ with $u=\bar{u}, R=2 / 3 \alpha, a=-1, b=1, t_{0}=0, M_{2} \simeq \bar{\Lambda}\left(Q_{\alpha}\right) / \alpha^{2}$.

By (5.10) and (5.12), we can apply Bombieri's lemma to the family of rectangles $R^{-}(\varrho)$ with $\mu=\alpha^{-2} \bar{\Lambda}\left(Q_{\alpha}\left(x_{0}\right)\right)+\alpha^{2} / \bar{\lambda}\left(Q_{\alpha}\left(x_{0}\right)\right), M=1 / \mu$ and $f=\mathrm{e}^{-\mathrm{M}_{2}+V(0)} \bar{u}$, obtaining

$$
\text { ess } \sup _{R^{-}(1 / 2)} f \leq C \exp \left\{c\left[\alpha^{-2} \bar{\Lambda}\left(Q_{\alpha}\right)+\alpha^{2} / \bar{\lambda}\left(Q_{\alpha}\right)\right]\right\},
$$

and this implies that

(5.13) $\operatorname{ess} \sup _{R^{-}(1 / 2)} \bar{u} \leq C \exp \left\{c\left[\alpha^{-2} \bar{\Lambda}\left(Q\left(x_{0}, \alpha\right)\right)+\alpha^{2} / \bar{\lambda}\left(Q\left(x_{0}, \alpha\right)\right)\right]-V(0)\right\}$.

Also, by (5.11) and (5.12), we can apply Bombieri's lemma to the family of rectangles $R^{+}(\varrho), f=\mathrm{e}^{-M_{2}-V(0)} \bar{u}^{-1}$, with $\mu, M, M_{2}$ and $V(0)$ as before, and we obtain 


$$
\text { ess } \sup _{R^{+}(1 / 2)} f \leq C \exp \left\{c\left[\alpha^{-2} \bar{\Lambda}\left(Q_{\alpha}\right)+\alpha^{2} / \bar{\lambda}\left(Q_{\alpha}\right)\right]\right\},
$$

which implies that

$$
\mathrm{e}^{-V(0)} \leq C e^{c\left[\alpha^{-2} \bar{\Lambda}\left(Q\left(x_{0}, \alpha\right)\right)+\alpha^{2} / \bar{\lambda}\left(Q\left(x_{0}, \alpha\right)\right) \mid\right.} \operatorname{ess}_{\inf _{R^{+}(1 / 2)} \bar{u} .}
$$

Combining (5.13) and (5.14) it follows that

$$
\text { ess } \sup _{R^{-}(1 / 2)} \bar{u} \leq c_{1} e^{c\left[\alpha^{-2} \bar{\Lambda}\left(Q\left(x_{0}, \alpha\right)\right)+\alpha^{2} / \bar{\lambda}\left(Q\left(x_{0}, \alpha\right)\right)\right]} \operatorname{ess} \inf _{R^{+}(1 / 2)} \bar{u} \text {. }
$$

Since, $T\left(R^{-}(1 / 2)\right)=R^{-}, T\left(R^{+}(1 / 2)\right)=R^{+}$and $\alpha^{-2} \bar{\Lambda}\left(Q_{\alpha}\right)+\alpha^{2} / \bar{\lambda}\left(Q_{\alpha}\right)=$ $\alpha^{-2} \beta \Lambda\left(Q_{\alpha}\right)+\alpha^{2} \beta^{-1} / \lambda\left(Q_{\alpha}\right)$, Theorem A follows.

REMARK: Using the equivalence between $d$ and $\delta$ we can prove the following analogues of Theorem $\mathrm{A}$ and $\mathrm{B}$ for the metric $d$.

Theorem $\mathbf{A}^{\prime}$ : Assume (i), (ii), (iii) of Theorem A. If $u$ is a non-negative solution of (1.1) in the cylinder $R=S\left(x_{0}, \alpha a^{2}\right) \times\left(t_{0}-\beta, t_{0}+\beta\right)$, then

ess $\sup _{R^{-}} u \leq c_{1} \exp \left\{c_{2}\left[\alpha^{-2} \beta \wedge\left(S\left(x_{0}, \alpha\right)\right)+\alpha^{2} \beta^{-1} \lambda\left(S\left(x_{0}, \alpha\right)\right)^{-1}\right]\right\} \operatorname{ess~inf}_{R^{+}} u$ where $R^{-}=S\left(x_{0}, \alpha / 2\right) \times\left(t_{0}-3 \beta / 4, t_{0}-\beta / 4\right), R^{+}=S\left(x_{0}, \alpha / 2\right) \times\left(t_{0}+\beta / 4\right.$, $\left.t_{0}+\beta\right), \Lambda(S)=w_{2}(S) / v(S)$ and $\lambda(S)=w_{1}(S) / v(S)$ for a $d$-ball $S$. Here the constants $c_{1}, c_{2}$ depend only on the constants which arise in (i), (ii), (iii).

Theorem B': Assume hypothesis (i), (ii), (iii) of Theorem A hold. Let $0<p<\infty, \alpha, \beta>0, \alpha / 2<\alpha^{\prime}<\alpha, \beta / 2<\beta^{\prime}<\beta$ and let $S\left(x_{0}, \alpha\right)=S$, $S\left(x_{0}, \alpha^{\prime}\right)=S^{\prime}$ and $R(\alpha, \beta)=S \times\left(t_{0}-\beta, t_{0}+\beta\right), R_{+}^{\prime}(\alpha, \beta)=S^{\prime} \times\left(t_{0}-\beta^{\prime}\right.$, $\left.t_{0}+\beta\right)$. If $u$ is a solution of $(1.1)$ in $R\left(a^{2} \alpha, \beta\right)$, then $u$ is bounded in $R_{+}^{\prime}(\alpha, \beta)$ and

ess $\sup _{R^{\prime}(\alpha, \beta)}|u|^{p} \leq$

$D\left(\alpha^{2} \beta^{-1} \lambda(S)^{-1}+1\right)^{1 /(h-1)}\left(\alpha^{-2} \beta \Lambda(S)+1\right)^{h /(h-1)} \iint_{R\left(a^{2} \alpha, \beta\right)}|u|^{p}\left(\alpha^{-2} \beta w_{2}+v\right) d x d t$ where $D$ is as in Theorem $B$, and $C=c \frac{\alpha^{2+b} \beta}{\left(\alpha-\alpha^{\prime}\right)^{2+b}\left(\beta-\beta^{\prime}\right)}$. Here $h>1$, constants which are independent of $u, p, \alpha, \alpha^{\prime}, \beta, \beta^{\prime}$.

\section{Acknowledgments}

I wish to thank Prof. Richard Wheeden for having suggested this problem, as well as for his generous advice throughout the work. 


\section{References}

[AS] D. G. Aronson and J. Serrin, Local behavior of solutions of quasilinear parabolic equations, Arch Rat. Mech. Anal. 25 (1967), 81-122.

[CW1] S. Chanillo and R. Wheeden, Harnack's inequality and mean value inequalities for solutions of degenerate elliptic equations, Comm. P.D.E. 11 (1986), 1111-1134.

[CW2] S. Chanillo and R. Wheeden, Weighted Poincaré's and Sobolev inequalities for the Peano maximal function, Amer. J. Math. 107 (1985), 1191-1226.

[CoW] R. Coifman and G. Weiss, Analyse harmonique non-commutative sur certain espaces homogènes, Springer-Verlag, 1971.

[CS F. Chiarenza and R. Serapioni, A Harnack inequality for degenerate parabolic equations, Comm. P.D.E. 9 (1984), 719-749.

[C] A. P. Calderón, Inequalities for the maximal function relative to a metric, Studia Math. 57 (1976), 297-306.

[CF $\quad$ R. R. Coifman and C. Fefferman, Weighted norm inequalities for maximal functions and singular integrals, Studia Math. 51 (1974), 241-250.

[FS] B. Franchi and R. Serapioni, Pointwise estimates for a class of strongly degerate elliptic operators: a geometrical approach, Ann. Scuola Norm. Sup. Pisa 14 (1987), 527-568.

[FL1] B. Franchi and E. Lanconelli, Hölder regularity for a class of linear non uniformily elliptic operators with measurable coefficients, Ann. Scuola Norm. Sup. Pisa 10 (1983), 523-541.

[FL2] B. Franchi and E. Lanconelli, Une metrique associée à une classe d'operateurs elliptiques dégénérés, Rend. Sem. Mat. Univ. Politec. Torino (1983), 105-114.

[GW1] C. Gutiérrez and R. Wheeden, Sobolev interpolation inequalities with weights, Trans. Amer. Math. Soc., to appear.

GW2 C. Gutiérrez and R. Wheeden, Mean value and Harnack inequalities for degenerate parabolic equations, Colloquium Math.

[GT] Gilbarg and N. S. Trudinger, Elliptic Partial Equations of Second Order, second edition, Springer-Verlag, 1977.

[J] D. Jerison, The Poincaré inequality for vector fields satisfying Hormander's condition, Duke Math. J. 53 (1986), 503-523.

[SW] E. Sawyer and R. Wheeden, Weighted inequalities for fractional integrals in Euclidean and homogeneous spaces, to appear.

Recibido: 20 de julio de 1990.

José C. Fernandes

Rutgers University

New Brunswick, NJ, 08903

Current Address:

Universidade de São Paulo

Caixa Postal 20570

São Paulo, SP, 01498

Brasil 